\title{
Symbol Error Probability Analysis of DFrFT-Based OFDM Systems With CFO and STO in Frequency Selective Rayleigh Fading Channels
}

\author{
Atul Kumar and Maurizio Magarini, Member, IEEE
}

\begin{abstract}
In this paper, we derive exact symbol error probability (SEP) expressions for orthogonal frequency-division multiplexing (OFDM) based on discrete fractional Fourier transform (DFrFT) when symbol timing offset (STO) and carrier frequency offset (CFO) are present together. Closed form expressions are derived for binary phase shift keying, quadrature phase shift keying, and 16-ary quadrature amplitude modulation in the case of transmission over a frequency selective Rayleigh fading channel. As it is well known, in OFDM the presence of CFO destroys the orthogonality between the sub-carriers, generating inter-carrier interference (ICI), while the presence of STO introduces intersymbol interference, between successive symbols, and phase rotations in frequency domain, within the same symbol. It is well recognized that an OFDM system based on DFrFT is more robust to ICI introduced by CFO than that based on discrete Fourier transform (DFT). Motivated by this, the combined effect of CFO and STO on the SEP of a DFrFT-based OFDM system is analyzed. Our results show that the DFrFT-based OFDM system outperforms that based on the DFT for different values of the DFrFT "angle parameter." Monte Carlo simulations are used to demonstrate the correctness of the derived analytical expressions.
\end{abstract}

Index Terms-Error rate analysis, sampling-type FrFT, frequency selective fading, orthogonal frequency-division multiplexing (OFDM), symbol timing offset, carrier frequency offset.

\section{INTRODUCTION}

$\mathbf{I}$ $\mathrm{N}$ the recent years discrete fractional Fourier transform (DFrFT) has been proposed in many fields of digital signal processing as a practical means of performing time-frequency analysis [1], [2]. In the context of wireless communications, the use of DFrFT was proposed in place of discrete Fourier transform (DFT) as multi-carrier modulation technique for orthogonal frequency-division multiplexing (OFDM). The advantages of DFrFT over DFT in OFDM systems for transmission over doubly selective channels, i.e., channels that are selective both in time and in frequency, were originally delineated in [3].

As is well known, the main advantages of OFDM are channel estimation, low-complexity equalization, efficient hardware implementation, and easy realization of MIMO transmission.

Manuscript received July 4, 2018; revised October 8, 2018 and October 28, 2018; accepted November 1, 2018. Date of publication November 14, 2018; date of current version January 15, 2019. The review of this paper was coordinated by Prof. G. Gui. (Corresponding author: Atul Kumar.)

The authors are with the Dipartimento di Elettronica, Informazione e Bioingegneria, Politecnico di Milano, Milan 20133, Italy (e-mail: atul.kumar@ polimi.it; maurizio.magarini@polimi.it).
Due to all these supportive properties, the DFT-based OFDM system has been adopted by many standards, such as wireless IEEE $802.11 \mathrm{a} / \mathrm{g} / \mathrm{n}$, local area networks, i.e., wireless metropolitan area networks, i.e., IEEE802.16a, terrestrial digital video broadcasting systems, and wireless cellular networks, i.e., 4th generation (4G) Long Term Evolution and 5th generation (5G) New Radio (NR) [4]-[7]. Concerning with 5G-NR, different variants of OFDM, i.e., DFT-spread OFDM, filtered OFDM, and windowed-OFDM, will play a key role in the scenarios categorized in the 2020 time frame by International Telecommunication Union, which are defined by the following three main groups [4]: i) enhanced mobile broadband (eMBB) [8]; ii) massive machine type communication (mMTC) [9]; iii) ultrareliable and low latency communications (URLLC)[10]. Also, many advanced technologies have been introduced to meet the different requirements arising from the three groups, such as, massive multiple input multiple output (MIMO) [11] and millimeter-wave (mmWave) communication [12].

Although OFDM has several advantages, it also presents some disadvantages that are mainly introduced by synchronization issues like high sensitivity to symbol timing offset (STO) and carrier frequency offset (CFO) [13]. CFO is due to either mismatching of transmitter and receiver oscillators frequencies or Doppler spread while STO is caused by the discrepancy between the current sampling phase and the optimal one. The presence of CFO destroys the orthogonality among sub-carriers, which generates inter-carrier interference (ICI) [14]. The presence of STO introduces phase rotations in frequency domain, within the same symbol, and intersymbol interference (ISI), between successive symbols [13].

There are many synchronization techniques available in the literature for estimating and compensating offsets in OFDM systems [15]. However, in the presence of oscillator drifts and timevarying Doppler shifts, residual CFO and STO are still present in the received signal after the application of synchronization algorithms. In order to improve the robustness to residual $\mathrm{CFO}$, an OFDM system based on the use DFrFT was proposed by Martone in [3]. This system is analogous to the conventional OFDM one with the difference that DFT and inverse DFT (IDFT) are replaced by DFrFT and inverse DFrFT (IDFrFT), respectively. As observed by Martone, in case of a time-varying frequency selective fading channel the entire conceptual framework of frequency-domain channel partitioning, exploited by DFT-based OFDM, loses its optimality. To counteract this drawback, Martone proposed the use of the DFrFT in OFDM where orthogonal signal bases of chirp function with rate $\cot (\alpha)$, being $\alpha$ the 'angle parameter', are used in place of the orthogonal complex exponentials of DFT in the presence of Doppler 
effect. The main advantage of using orthogonal chirp function consists in the control of the time-frequency partition of the channel. Here, as already observed in the existing literature, the same concept is used to mitigate the performance degradation introduced by the loss of orthogonality due to CFO. Moreover, concerning STO, it is here observed for the first time that DFrFT is also beneficial in terms of performance. This is demonstrated by quantifying its effect in an analytical expression of the term responsible for introducing ICI and ISI, which is part of novel contribution.

It is worth noting that the higher tolerance to CFO and STO of DFrFT-based OFDM systems is achieved without any increase in implementation complexity compared to efficient implementation of DFT, which is known as fast Fourier transform (FFT). However, in the literature several versions of DFrFT algorithms have been proposed [16]. The proposed versions belong to four different categories: eigenvector decomposition-based method [17], weighted summation-based method [18], linear combination-based method [19], and sampling-based method [20]. As shown in Table I of [16], the complexity mainly depends on the constraints set on the implementations of different DFrFT algorithms. By following [16], we observe that when the block of length $N$ is a power of 2 , the run time complexity of DFrFT computation is in the order of $O\left(N^{2}\right)$ for the eigenvector decomposition and the weighted summation-based methods. In contrast, the complexity of sampling and linear-based methods is in the order of $O\left(N \log _{2} N\right)$, which is the same as that of FFT. For the sampling-based method this complexity is achieved by means of the Pei's algorithm [21]. Concerning with the use of DFrFT algorithm in OFDM, the most important property that must be satisfied is the reversibility in the IDFrFT computation. For this reason, we focus on the closed-form type form of sampling-based method that ensures such a property [22]. Note that, a further reduction in the complexity of DFT can be achieved via a novel sub-linear algorithm, named sparse Fourier transform, developed by Haitham et al. [23]. When the input data have a large size with a sparse spectrum, this algorithm reduces the complexity of DFT to $O\left(\log _{2} N \cdot \sqrt{k N \log _{2} N}\right)$, where $k$ stands for the number of large coefficients in the frequency domain. Similarly, Liu et al. [22] proposed the sparse DFrFT to achieve fast computation. These recent results about the low complexity implementation of DFrFT are the main motivation towards hardware implementations as, for example, those described in [24].

An important measure to evaluate the robustness of DFrFT with respect to residual CFO and STO is represented by the error rate performance. Closed form expressions for the symbol error probability (SEP) of a DFrFT-based OFDM system for frequency selective fading channels in presence of CFO were derived by many authors [25]-[29]. In [25], an exact bit error probability (BEP) formula was derived for binary phase shift keying (BPSK) transmission over the Rayleigh fading channel. For the same modulation and channel model, the authors in [26] analyzed BEP for the weighted-type DFrFT impaired by CFO. Always considering BPSK, in [27] the exact BEP of the hyperbolic DFrFT was derived for transmission over the Rician fading channel. In [28], the authors extended the work of [25] to the case of quadrature phase shift keying (QPSK) and derived an exact SEP expression for frequency selective Rayleigh fading channel. Finally, the analysis done in [29] extended SEP performance evaluation to the transmission over a Nakagami- $m$ fading channel both for BPSK and for QPSK modulation formats.

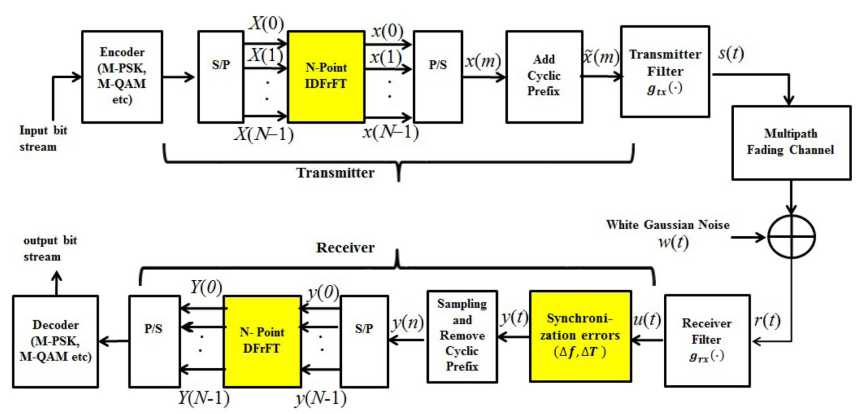

Fig. 1. DFrFT-based OFDM system model.

However, since it happens that CFO and STO are present simultaneously, a more complete picture is obtained by analyzing the performance when their effect is considered together instead of dealing only with CFO.

The main contributions of this paper are:

1) Definition of a DFrFT-based OFDM system model for a generalized multipath channel that includes both $\mathrm{CFO}$ and STO effects.

2) Derivation of exact SEP expressions for a DFrFT-based OFDM system with simultaneous presence of $\mathrm{CFO}$ and STO. The analytical closed form expressions are given for BPSK, QPSK, and 16-QAM modulation formats in case of transmission over a frequency selective Rayleigh fading channel.

3) Derivation of the closed form expression of the optimal DFrFT angle parameter $\alpha$ as a function of CFO.

The remaining sections are organized as follows. Section II describes the DFrFT-based OFDM system model in presence of CFO and STO. In Section III the derivations of exact SEP analytical expressions for BPSK, QPSK, and 16-QAM are given in case of transmission over a frequency selective Rayleigh channel. Possible extension to the massive MIMO case is suggested in Section IV. In Section V the derivation of an optimal closed form expression for the DFrFT angle parameter is given. In order to confirm the analytical results obtained in Section III, a comparison with Monte Carlo simulations is reported in Section VI. Finally, conclusion is drawn in Section VII.

\section{SySTEM MODEL}

Figure 1 reports the block diagram of the complex baseband equivalent model of the DFrFT-based OFDM system. The transmitter includes the encoder block and the serial-to-parallel (S/P) converter block whose role is that of splitting the high data rate stream into a number of $N$ lower data rate parallel streams. The IDFrFT is computed on a block of $N$ information symbols. To overcome the effect of ISI, a cyclic prefix (CP) is inserted between successive OFDM symbols that consists of $N_{C P}$ samples, such that the length of the $\mathrm{CP}$ is at least equal to the memory of the channel measured in sampling intervals. Under this condition, the linear convolution between the transmitted sequence and the channel is converted to a circular convolution. The cyclically extended sequence is first sent at the input of a continuous-time pulse shaping filter and then transmitted through a time-varying multi-path fading channel. At the receiving side, a white Gaussian noise is added to the continuous time received signal and the resulting signal is filtered through the receiving pulse shaping filter and sampled. The $\mathrm{CP}$ is then removed and the resulting samples are $\mathrm{S} / \mathrm{P}$ converted and sent 
to the DFrFT block. The $N$ samples at the output of the DFrFT are equalized, again serialized, and finally decoded to produce an estimate of the originally transmitted data.

\section{A. Model of the Transmitted Signal}

The input bit stream is encoded into symbols taken from a given set of complex constellation points. The resulting $N$ complex symbols $X(k), k=0,1,2, \ldots, N-1$, are fed into the IDFrFT block, whose $m$ th output sample is written as

$$
x(m)=\sum_{k=0}^{N-1} X(k) F_{-\alpha}(m, k), m=0,1,2, \ldots, N-1,
$$

where $F_{-\alpha}(m, k)$ is the kernel defined as

$$
\begin{aligned}
F_{-\alpha}(m, k)= & \sqrt{\frac{\sin \alpha+j \cos \alpha}{N}} \\
& e^{\frac{-j m^{2} T^{2} \cot (\alpha)}{2}} e^{\frac{-j k^{2} u^{2} \cot (\alpha)}{2}} e^{\frac{j 2 \pi m}{N}},
\end{aligned}
$$

being $u$ the sampling interval in the fractional Fourier domain and $T$ the sampling interval in the time domain. The two sampling intervals are related as $u \times T=\frac{2 \pi|\sin (\alpha)|}{N}$. The angle between the fractional Fourier domain and the time domain is $\alpha=a \times \frac{\pi}{2}$, with $0 \leq a \leq 1$, where $a$ is a real number comprised between 0 and 1. For $\alpha=\pi / 2$, that is $a=1$, the DFrFT-based OFDM converts into DFT-based OFDM.

A CP of length $N_{C P}$ is appended at the beginning of the block of $N$ samples $x(m), m=0, \ldots, N-1$, given in (1). The resulting discrete-time sequence is then applied at the input of continuous-time baseband transmit filter with impulse response $g_{t x}(t)$ that, in the following, is assumed to be a square root raised cosine filter with unit energy. By following [30], when dealing with the presence of STO a suffix of length $N_{G}$ must be introduced in order to take into account of the effect, in each time instant $t$, of the $N_{G}$ symbols that precede and follow the actual one. Although in theory the length of $N_{G}$ for raised cosine impulse responses should be set to infinite, in a practical case we can assume for it a "finite" value, which depends on the considered roll-off parameter. The value of $N_{C P}$ must be chosen higher or equal to $L_{c h}-1$, where $L_{c h}$ is the length of the channel measured in sampling intervals. In general, one has $N_{C P} \geq N_{G}$. However, if it happens that $N_{C P} \leq N_{G}$ we must increase $N_{C P}$ to $N_{G}$. Therefore, we have $N_{C P}=\max \left(L_{c h}-\right.$ $\left.1, N_{G}\right)$. The resulting baseband complex equivalent expression of the the continuous-time OFDM signal is

$$
s(t)=\sum_{m=-N_{C P}}^{N-1+N_{G}} \tilde{x}(m) g_{t x}(t-m T), \quad 0 \leq t<T_{s},
$$

where $T_{s}=N T$ is the OFDM symbol interval and

$$
\tilde{x}(m)= \begin{cases}x(N+m), & m=-N_{C P}, \ldots,-1, \\ x(m), & m=0, \ldots, N-1, \\ x(m-N-1), & m=N+1, \ldots, N+N_{G} .\end{cases}
$$

\section{B. Channel Model}

The $L$-path tapped delay line baseband equivalent channel model defined in [31] is here considered. According to this model, the impulse response of the time-varying multi-path fading channel is defined as

$$
h(t)=\sum_{l=0}^{L-1} h_{l}(t) \delta\left(t-\tau_{l} T\right),
$$

where $h_{l}(t)$ is the complex amplitude, or tap coefficient, at time $t$ of the $l$ th path associated with the propagation delay $\tau_{l} T$, with $\tau_{l}$ being a real positive number, and $\delta(t)$ is the delta Dirac function. For the coefficients of the channel, we consider a model, where $h_{l}(t), l=0,1,2, \ldots, L-1$, are independent and identically distributed complex random variables with Rayleigh distributed amplitude, uniform distributed phase in $[0,2 \pi)$, and average power $\sigma_{l}^{2}=1 / L$. Therefore, $\sigma_{0}^{2}+\sigma_{1}^{2}+\cdots+\sigma_{L-1}^{2}=1$. In the following, we consider the case where $\tau_{l}=l$, which implies having delays at integer multiples of $T$ and, therefore, it results in $L_{c h}=L$.

\section{Model of the Received Signal With CFO and STO}

After transmission over the channel the received signal $r(t)$ is expressed as

$$
r(t)=\sum_{l=0}^{L-1} h_{l}(t) s(t-l T)+w(t)
$$

where $w(t)$ represents the complex additive white Gaussian noise (AWGN) at the receiver with power spectral density $N_{0}$. The signal in (6) is filtered by the receiving filter $g_{r x}(t)$, which is matched to $g_{t x}(t)$, to give

$$
u(t)=r(t) \circledast g_{r x}(t),
$$

where $\circledast$ denotes linear convolution. In case of perfect synchronization the only distortions are introduced by the multi-path channel and by the Gaussian noise. However, due to estimation errors, residual timing and frequency offsets exist. The continuous-time model of the received signal that includes their effects is

$$
y(t)=e^{j 2 \pi \Delta f t} u(t-\Delta T),
$$

where $\Delta f$ and $\Delta T$ denotes residual CFO and STO, respectively.

The received signal is sampled at time instants $t=n T$. After removal of the $\mathrm{CP}$, the resulting discrete-time signal model is

$$
\begin{aligned}
y(n T)= & e^{\frac{j 2 \pi \Delta \epsilon}{N}} \sum_{l=0}^{L-1} h_{l}(n T) \sum_{m=-N_{C P}}^{N-1+N_{G}} \tilde{x}(m) \\
& g(n T-m T-l T-\Delta T)+\tilde{w}(n T),
\end{aligned}
$$

where $\Delta \epsilon=\Delta f T_{s}$ is the residual CFO normalized to the subcarrier spacing $1 / T_{s}, \tilde{w}(n T)=w(n T-\Delta T)$ is the AWGN, and $g(t)=g_{t x}(t) \circledast g_{r x}(t)$, which is a raised cosine impulse response that satisfies the Nyquist condition for not having ISI such as

$$
g(t)=\frac{\sin \frac{\pi t}{T}}{\frac{\pi t}{T}} \cdot \frac{\cos \frac{\pi \rho t}{T}}{1-\left(\frac{2 \rho t}{T}\right)^{2}}
$$

with $\rho$ denoting the roll-off factor [31]. By omitting $T$, the fractionally Fourier domain signal after the DFrFT block is

$$
Y(q)=\sum_{n=0}^{N-1} y(n) F_{\alpha}(q, n)
$$


where $F_{\alpha}(q, n)$ is the kernel defined as

$$
F_{\alpha}(q, n)=\sqrt{\frac{\sin \alpha-j \cos \alpha}{N}} e^{\frac{j n^{2} T^{2} \cot (\alpha)}{2}} e^{\frac{j q^{2} u^{2} \cot (\alpha)}{2}} e^{\frac{-j 2 \pi n q}{N}} .
$$

Substituting the value of $y(n)$ given in (9) into (11) we get

$$
\begin{aligned}
& Y(q)=\sum_{n=0}^{N-1}\left\{e^{\frac{j 2 \pi \Delta \epsilon n}{N}} \sum_{l=0}^{L-1} h_{l}(n) \sum_{m=-N_{C} P}^{N-1+N_{G}} \sum_{k=0}^{N-1} X(k)\right. \\
& \left.F_{-\alpha}(m, k) g(n-m-l-\Delta \theta)+\tilde{w}(n)\right\} F_{\alpha}(q, n),
\end{aligned}
$$

where $\Delta \theta=\Delta T / T$ is the normalized residual fractional STO. By following [30], we apply the change of variable $n-m=$ $p+N_{C P}$ in (15) to get

$$
\begin{aligned}
Y(q)=\sum_{n=0}^{N-1}\left\{e^{\frac{j 2 \pi \Delta \epsilon n}{N}} \sum_{k=0}^{N-1} X(k) \sum_{p=n}^{n-N+1-N_{G}-N_{C P}} \sum_{l=0}^{L-1} h_{l}(n)\right. \\
g\left(p+N_{C P}-l-\Delta \theta\right) F_{-\alpha}\left(\left(n-p-N_{C P}\right)_{N}, k\right) \\
+\tilde{w}(n)\} F_{\alpha}(q, n) .
\end{aligned}
$$

Also, by using the same approximation of [30], where $g(t) \approx 0$ is assumed for $|t| \geq N_{G} T$, we obtain

$$
\begin{gathered}
Y(q)=\sum_{n=0}^{N-1}\left\{e^{\frac{j 2 \pi \Delta \epsilon n}{N}} \sum_{k=0}^{N-1} X(k) F_{-\alpha}(n, k) \sum_{l=0}^{L-1} h_{l}(n)\right. \\
\left.\sum_{m=-N_{G}}^{N_{C P}} g(m-l-\Delta \theta) e^{-j \frac{2 \pi}{N} k m}+\tilde{w}(n)\right\} F_{\alpha}(q, n) .
\end{gathered}
$$

In order to have a more compact notation, a matrix representation is adopted. Also, we make the assumption of block fading channel where the coefficients $h_{l}(n)$, with $l=0, \ldots, L-1$, remain constant for the entire duration of the OFDM symbol, i.e., $h_{l}(n)=h_{l}$. Let $\boldsymbol{X}=[X(0), X(1), \ldots, X(N-1)]^{T}$ be the $N \times 1$ vector of symbols applied at the input of the IDFrFT block to give the transmitted vector

$$
\boldsymbol{x}=\boldsymbol{F}_{-\alpha} \boldsymbol{X},
$$

where $\boldsymbol{F}_{-\alpha}$ is the $N \times N$ IDFrFT kernel matrix whose element $(m, k)$ is given in (2). Similarly, for $\tilde{x}(m), m=-N_{C P}, \ldots$, $N+N_{G}$, we define the vector

$$
\tilde{\boldsymbol{x}}=\left[\boldsymbol{x}^{T}\left(N-N_{C P}+1: N\right) \boldsymbol{x}^{T} \boldsymbol{x}^{T}\left(1: N_{G}\right)\right],
$$

where $\boldsymbol{x}(n: m)$ denotes the elements of vector $\boldsymbol{x}$ from $n$ to $m$ given by

$$
\boldsymbol{x}(n: m)=\boldsymbol{F}_{-\alpha}^{n: m, k}(n: m, k)
$$

being $\boldsymbol{F}_{-\alpha}^{n: m, k}$ the $(m-n) \times N$ matrix obtained by taking rows of $\boldsymbol{F}_{-\alpha}(n, k)$ from $n$ to $m$, with $m>n$. After some mathematical manipulation we can rewrite (18) as

$$
\tilde{\boldsymbol{x}}=\tilde{\boldsymbol{F}}_{-\alpha} \boldsymbol{X},
$$

where $\tilde{\boldsymbol{F}}_{-\alpha}=\left[\tilde{\boldsymbol{F}}_{-\alpha}^{\left(N-N_{C P}-1: N, k\right)} \boldsymbol{F}_{-\alpha} \boldsymbol{F}_{-\alpha}^{\left(1: N_{G}, k\right)}\right]$. With the introduced matrix notation, (9) is rewritten as the column vector

$$
\boldsymbol{y}=\boldsymbol{D}(\Delta \epsilon) \sum_{l=0}^{L-1} h_{l} \boldsymbol{G}_{\boldsymbol{l}}(\Delta \theta) \tilde{\boldsymbol{x}}+\tilde{\boldsymbol{w}}
$$

where $\boldsymbol{D}(\Delta \epsilon)=\operatorname{diag}\left(1, e^{\frac{j 2 \pi \Delta \epsilon}{N}}, \ldots, e^{\frac{j 2 \pi \Delta \epsilon(N-1)}{N}}\right)$ is the residual CFO matrix, with $\operatorname{diag}(\cdot)$ denoting a diagonal matrix, $\tilde{\boldsymbol{w}}=$ $[\tilde{w}(0), \ldots, \tilde{w}(N-1)]^{T}$ is the noise vector, and $\boldsymbol{G}_{l}(\Delta \theta)$ is the time domain STO matrix. The received signal in the fractionally Fourier domain is written as

$$
\boldsymbol{Y}=\boldsymbol{S}(\Delta \epsilon, \Delta \theta) \boldsymbol{H}_{d} \boldsymbol{X}+\tilde{\boldsymbol{W}}
$$

where $\boldsymbol{S}(\Delta \epsilon, \Delta \theta)=\boldsymbol{F}_{\alpha} \boldsymbol{D}(\Delta \epsilon) \boldsymbol{F}_{-\alpha} \tilde{\boldsymbol{G}}(\Delta \theta), \tilde{\boldsymbol{W}}=\boldsymbol{F}_{\alpha} \tilde{\boldsymbol{w}}$ is the noise vector, and $\tilde{G}(\Delta \theta)$ is a diagonal matrix represented as

$$
\begin{aligned}
& \tilde{\boldsymbol{G}}(\Delta \theta)=\operatorname{diag}\left\{\sum_{m=-N_{C}}^{N_{G}} g(m-\Delta \theta) e^{\frac{-j 2 \pi m 0}{N}}, \sum_{m=-N_{C P}}^{N_{G}}\right. \\
& \left.g(m-\Delta \theta) e^{\frac{-j 2 \pi m 1}{N}}, \ldots, \sum_{m=-N_{C P}}^{N_{G}} g(m-\Delta \theta) e^{\frac{-j 2 \pi m(N-1)}{N}}\right\} .
\end{aligned}
$$

In (21), $\boldsymbol{H}_{d}=\operatorname{diag}(\boldsymbol{\beta})$, with $\boldsymbol{\beta}=\boldsymbol{F}_{1: L} \boldsymbol{h}$, where $\boldsymbol{h}=[h(0)$, $\ldots, h(L-1)]^{T}$ is the time-domain vector of channel coefficients and $\boldsymbol{F}_{1: L}$ corresponds to the first $L$ columns of the $N \times N$ DFT matrix. The $k$ th sub-carrier of the received signal vector given in (21) is given in (22), shown at the bottom of this page, where $X(k)$ is the transmitted complex data symbol over the $k$ th sub-carrier, $\beta(k)$ is the channel frequency response for the $k$ th sub-carrier, $\tilde{w}(k)$ is the complex AWGN with zero mean and variance per dimension $\sigma^{2}=N_{0} / 2$, and

$$
\begin{aligned}
S(l, k)= & \frac{1}{N} \sum_{n=0}^{N-1} e^{j \frac{2 \pi \Delta \epsilon n}{N}} \sum_{m=0}^{N-1} e^{-j \frac{\left(m^{2}-n^{2}\right) T^{2} \cot (\alpha)}{2}} \\
& e^{j \frac{\left(l^{2}-k^{2}\right) u^{2} \cot (\alpha)}{2}} e^{j \frac{2 \pi(m k-n l)}{N}} \cdot b(l+k),
\end{aligned}
$$

where $b(n)=[\tilde{\boldsymbol{G}}(\Delta \theta)]_{n, n}$.

\section{Symbol ERror Probability ANALYSis}

In this section we present the exact SEP analysis of DFrFTbased OFDM system in presence of CFO and STO for BPSK, QPSK, and 16-QAM modulation formats when transmission takes place over a frequency selective Rayleigh fading channel.

$$
Y(k)=\underbrace{\underbrace{X(k) S(0, k) \beta(k)}_{\begin{array}{c}
\text { Desired signal } \\
\text { on the } k \text { th sub-carrier }
\end{array}}+\underbrace{\sum_{q=0, q \neq k}^{N-1} X(q) S\left(<q-k>_{N}, k\right) \beta(q)}_{\begin{array}{c}
\text { ICI and ISI terms } \\
\text { due to sub-carriers other than } k
\end{array}}}_{R(k)}+\tilde{w}(k), \quad k=0, \ldots, N-1,
$$




\section{A. BPSK}

In the case of BPSK modulation scheme, symbols are drawn from the set $\{ \pm 1\}$. As in [32], we evaluate the performance on the $k$ th sub-carrier when $X(k)=+1$. With this assumption the received signal on the $k$ th sub-carrier is expressed as

$$
Y(k)=S(0, k) \beta(k)+\sum_{q=0, q \neq k}^{N-1} X(q) S(q, k) \beta(q)+\tilde{w}(k) .
$$

The equalized signal on the $k$ th sub-carrier is given by

$$
\begin{aligned}
& \beta^{*}(k) Y(k)=S(0, k)|\beta(k)|^{2} \\
& \quad+\sum_{q=0, q \neq k}^{N-1} X(q) S(q, k) \beta(q) \beta^{*}(k)+\tilde{w}(k) \beta^{*}(k),
\end{aligned}
$$

where $^{*}$ denotes complex conjugation. It is worth noting that, when STO is also present, in contrast to the case of CFO only, each sub-carrier is characterized by a different SEP [30]. The SEP on the $k$ th sub-carrier is

$$
P_{s}\left(\xi_{k}\right)=\int_{\boldsymbol{\beta}} P_{s}\left(\xi_{k} \mid \boldsymbol{\beta}\right) p_{\boldsymbol{B}}(\boldsymbol{\beta}) d \boldsymbol{\beta}
$$

where $P_{s}\left(\xi_{k} \mid \boldsymbol{\beta}\right)$ is the SEP conditioned to a given $\boldsymbol{\beta}$, this latter being described by the $N$-dimensional joint probability density function (PDF) $p_{\boldsymbol{B}}(\boldsymbol{\beta})$. Since the evaluation of the above multidimensional integral is difficult, a simplification can be obtained by using the following factorization

$$
p_{\boldsymbol{B}}(\boldsymbol{\beta})=p_{\boldsymbol{\beta}_{k} \mid \beta(k)}\left(\boldsymbol{\beta}_{k} \mid \beta(k)\right) p_{\beta(k)}(\beta(k)),
$$

where $\boldsymbol{\beta}_{k}=[\boldsymbol{\beta}(0: k-1) \boldsymbol{\beta}(k+1: N-1)]^{T}, p_{\beta(k)}(\beta(k))$ is the one dimensional PDF of $\beta(k)$, and $p_{\boldsymbol{\beta}_{k} \mid \beta(k)}\left(\boldsymbol{\beta}_{k} \mid \beta(k)\right)$ is the conditional PDF of $\boldsymbol{\beta}_{k}$ given $\beta(k)$. By replacing (27) in (26) we obtain

$$
P_{s}\left(\xi_{k}\right)=\int_{0}^{\infty} P_{s}\left(\xi_{k} \mid \beta(k)\right) p_{\beta(k)}(\beta(k)) d \beta(k),
$$

where

$$
P_{s}\left(\xi_{k} \mid \beta(k)\right)=\int_{\boldsymbol{\beta}_{k}} P_{s}\left(\xi_{k} \mid \beta(k), \boldsymbol{\beta}_{k}\right) p_{\boldsymbol{\beta}_{k} \mid \beta(k)}\left(\boldsymbol{\beta}_{k} \mid \beta(k)\right) d \boldsymbol{\beta}_{k} .
$$

The expression of $P_{s}\left(\xi_{k} \mid \beta(k), \boldsymbol{\beta}_{k}\right)$ can be obtained by computing the conditional characteristic function $(\mathrm{CHF})$ of the received signal approach described in [28]. The considered conditional $\mathrm{CHF}$ of the real part of the equalized received signal given $\beta(k), \boldsymbol{\beta}_{k}$, defined as $\Re\left[\beta^{*}(k) Y(k) \mid \beta(k), \boldsymbol{\beta}_{k}\right]$ in (25), is

$$
\varphi_{\Re\left[\beta^{*}(k) Y(k) \mid \beta(k), \boldsymbol{\beta}_{k}\right]}(\omega)=E\left[e^{j w \Re\left[\beta^{*}(k) Y(k)\right]} \mid \beta(k), \boldsymbol{\beta}_{k}\right],
$$

where $\Re[\cdot]$ denotes the real part and $E[\cdot]$ denotes the statistical expectation. Substituting (25) in (30) and averaging over the AWGN we get

$$
\begin{gathered}
\varphi_{\Re\left[\beta^{*}(k) Y(k) \mid \beta(k), \boldsymbol{\beta}_{k}\right]}(\omega)=e^{j w \Re\left[S(0, k)|\beta(k)|^{2}\right]} \\
\prod_{q=0, q \neq k}^{N-1} E\left[e^{j \omega \Re\left[X(k) S(q, k) \beta(q) \beta^{*}(k)\right]}\right] \cdot e^{-\frac{\omega^{2} \sigma_{\beta}^{2}}{2}},
\end{gathered}
$$

where $\sigma_{\beta}^{2}=|\beta(k)|^{2} \sigma^{2}$. By computing the average over all the possible combinations of symbols and by using the trigonomet- ric identity given by [32, eq. (3)], from (31) we get

$$
\begin{aligned}
& \varphi_{\Re\left[\beta^{*}(k) Y(k) \mid \beta(k), \boldsymbol{\beta}_{k}\right]}(\omega)=e^{j \omega \Re\left[S(0, k)|\beta(k)|^{2}\right]} \cdot e^{-\frac{\omega^{2} \sigma_{\beta}^{2}}{2}} \\
& \frac{1}{2^{N-1}} \sum_{q=0, q \neq k}^{2^{N-2}} e^{j \omega \Re\left[\beta^{*}(k) \boldsymbol{e}_{q}^{T} \boldsymbol{\Lambda} \boldsymbol{\beta}_{k}\right]}+e^{-j \omega \Re\left[\beta^{*}(k) \boldsymbol{e}_{q}^{T} \boldsymbol{\Lambda} \boldsymbol{\beta}_{k}\right]},
\end{aligned}
$$

where $\boldsymbol{\Lambda}=\operatorname{diag}(S(1, k), S(2, k), \ldots, S(N-1, k))$ and $\boldsymbol{e}_{q}$ is an $N \times 1$ vector corresponding to the binary codeword of the number $2^{N-1}-q$, in which zeros have been substituted with $-1 \mathrm{~s}$. After some straightforward mathematical passages, (32) can be rewritten as

$$
\begin{aligned}
& \varphi_{\Re\left[\beta^{*}(k) Y(k) \mid \beta(k), \boldsymbol{\beta}_{k}\right]}(\omega)=\frac{1}{2^{N-1}} \cdot e^{-\frac{\omega^{2} \sigma_{\beta}^{2}}{2}} \\
& \sum_{q=0}^{2^{N-2}-1} e^{j \omega\left(\Re\left[S(0, k)|\beta(k)|^{2}\right]+\Re\left[\beta^{*}(k) \boldsymbol{e}_{q}^{T} \boldsymbol{\Lambda} \boldsymbol{\beta}_{k}\right]\right)} \\
& +\sum_{q=0}^{2^{N-2}-1} e^{j \omega\left(\Re\left[S(0, k)|\beta(k)|^{2}\right]-\Re\left[\beta^{*}(k) \boldsymbol{e}_{q}^{T} \boldsymbol{\Lambda} \boldsymbol{\beta}_{k}\right]\right) .}
\end{aligned}
$$

It can be observed that (33) represents the $\mathrm{CHF}$ of a mixture of Gaussian densities [32]. Now, an error will occur only if $\Re\left[\beta^{*}(k) Y(k) \mid \beta(k) \boldsymbol{\beta}_{k}\right]<0$. The probability of this event can be computed as

$$
P_{s}\left(\xi_{k} \mid \beta(k), \boldsymbol{\beta}_{k}\right)=\int_{-\infty}^{0} f_{\Re\left[\beta^{*}(k) Y(k) \mid \beta(k), \boldsymbol{\beta}_{k}\right]}(x) d x,
$$

where $f_{\Re\left[\beta^{*}(k) Y(k) \mid \beta(k), \boldsymbol{\beta}_{k}\right]}(\cdot)$ is the conditional PDF obtained from the inverse Fourier transform (IFT) of the conditional CHF given in (33). The solution of (34) gives the following expression of the resulting conditional SEP

$$
\begin{aligned}
& P_{s}\left(\xi_{k} \mid \beta(k), \boldsymbol{\beta}_{k}\right)=\frac{1}{2^{N-1}} \sum_{q=0}^{2^{N-2}-1} \\
& Q\left(\frac{|\beta(k)|^{2} \Re[S(0, k)]+b_{q}^{k}}{\sigma_{\beta}}\right)+Q\left(\frac{|\beta(k)|^{2} \Re[S(0, k)]-b_{q}^{k}}{\sigma_{\beta}}\right),
\end{aligned}
$$

where $b_{q}^{k}=\Re\left[\beta^{*}(k) \boldsymbol{e}_{q}^{T} \boldsymbol{\Lambda} \boldsymbol{\beta}_{k}\right]$. The expression of $P_{s}\left(\xi_{k} \mid \beta(k)\right)$ can now be obtained by substituting (35) in (29) and averaging over the conditional Gaussian PDF $p_{\boldsymbol{\beta}_{k} \mid \beta(k)}\left(\boldsymbol{\beta}_{k} \mid \beta(k)\right)$, with mean $E\left[\boldsymbol{\beta}_{k} \mid \beta(k)\right]$ and covariance $C_{\boldsymbol{\beta}_{k} \mid \beta(k)}[32$, eq. (27)]. The resulting expression is

$$
\begin{aligned}
P_{s}\left(\xi_{k} \mid \beta(k)\right)= & \frac{1}{2^{N-1}} \sum_{q=0}^{2^{N-2}-1} Q\left(\frac{|\beta(k)|\left(\Re[S(0, k)]+z_{q}^{k}\right)}{\sigma \sqrt{1+\frac{a_{q}^{k}}{2 \sigma^{2}}}}\right) \\
& Q\left(\frac{|\beta(k)|\left(\Re[S(0, k)]-z_{q}^{k}\right)}{\sigma \sqrt{1+\frac{a_{q}^{k}}{2 \sigma^{2}}}}\right)
\end{aligned}
$$

where $z_{q}^{k}$ and $a_{q}^{k}$ are related to the mean and to the variance of the conditional random variable $\left(b_{q}^{k} \mid \beta(k)\right)$ as [25]

$$
z_{q}^{k}=\frac{E\left[b_{q}^{k} \mid \beta(k)\right]}{|\beta(k)|^{2}}=C_{\beta(k) \beta(k)}^{-1} \Re\left[\left(\boldsymbol{e}_{q}^{T} \boldsymbol{\Lambda}\right) C_{\boldsymbol{\beta}_{k} \beta(k)}\right]
$$


and

$$
a_{q}^{k}=2 \frac{\operatorname{var}\left[b_{q}^{k} \mid \beta(k)\right]}{|\beta(k)|^{2}}=\left(\boldsymbol{e}_{q}^{T} \boldsymbol{\Lambda}\right) C_{\boldsymbol{\beta}_{k} \mid \beta(k)}\left(\boldsymbol{e}_{q}^{T} \boldsymbol{\Lambda}\right)^{H},
$$

respectively. In (37) and (38) we have $C_{\boldsymbol{\beta}_{k} \beta(k)}=E\left[\boldsymbol{\beta}_{k} \beta^{*}(k)\right]$, $C_{\left(\boldsymbol{\beta}_{k} \mid \beta(k)\right)}=C_{\boldsymbol{\beta}_{k} \boldsymbol{\beta}_{k}}-C_{\beta(k) \beta(k)}^{-1} C_{\boldsymbol{\beta}_{k} \beta(k)} C_{\boldsymbol{\beta}_{k} \beta(k)}^{H}, C_{\left(\boldsymbol{\beta}_{k} \boldsymbol{\beta}_{k}\right)}=$ $\boldsymbol{F}_{1: L} \boldsymbol{h} \boldsymbol{F}_{1: L}^{H}$, being $(\cdot)^{H}$ the Hermitian transpose conjugation. In order to calculate the overall SEP we first introduce the change of variable $\gamma_{k}=2|\beta(k)|^{2} / \sigma^{2}$, which allows us to express (36) as a function of the instantaneous signal-to-noise ratio (SNR) $\gamma_{k}$ on the $k$ th sub-carrier, and then we average (28) over all the sub-carriers as

$$
P_{s}(\xi)=\frac{1}{N} \sum_{k=0}^{N-1} P_{s}\left(\xi_{k}\right)=\frac{1}{N} \sum_{k=0}^{N-1} \int_{0}^{\infty} P_{s}\left(\xi_{k} \mid \gamma_{k}\right) p_{\gamma_{k}}\left(\gamma_{k}\right) d \gamma_{k},
$$

where $p_{\gamma_{k}}\left(\gamma_{k}\right)=\bar{\gamma}_{k}^{-1} e^{\frac{\gamma_{k}}{\gamma_{k}}}$ is the PDF of Rayleigh fading with average $\mathrm{SNR} \bar{\gamma}_{k}=2 E\left[|\beta(k)|^{2}\right] / \sigma^{2}$.

In what follows we adopt the polar form definition given in [31] for the $Q$-function in (36). The overall SEP is computed by applying the moment generating function (MGF) approach in (39) as

$$
\begin{aligned}
& P_{s}(\xi)=\frac{1}{\pi N 2^{N-1}} \sum_{q=0}^{2^{N}-2} \sum_{k=0}^{N-1} \int_{0}^{\frac{\pi}{2}} \\
& \{\underbrace{\int_{0}^{\infty} \exp \left(-\frac{\gamma_{k}\left(\Re[S(0, k)]+z_{q}^{k}\right)^{2}}{\left(1+\frac{a_{q}^{k}}{2 \sigma^{2}}\right) \sin ^{2} \theta}\right) p_{\gamma_{k}}\left(\gamma_{k}\right) d \gamma_{k}}_{M_{\gamma_{k}}\left(s_{1}\right)} \\
& +\underbrace{\int_{0}^{\infty} \exp \left(-\frac{\gamma_{k}\left(\Re[S(0, k)]-z_{q}^{k}\right)^{2}}{\left(1+\frac{a_{q}^{k}}{2 \sigma^{2}}\right) \sin ^{2} \theta}\right) p_{\gamma_{k}}\left(\gamma_{k}\right) d \gamma_{k}}_{M_{\gamma_{k}}\left(s_{2}\right)}\}
\end{aligned}
$$

where $M_{\gamma_{k}}(s)$ is used to denote the scaled Laplace transform of $p_{\gamma_{k}}\left(\gamma_{k}\right)$, which is known as MGF. The analytical expression of the MGF for the Rayleigh distribution is given by

$$
M_{\gamma_{k}}(s)=\left(1-s \bar{\gamma}_{k}\right)^{-1} \text {. }
$$

The computation of $P_{s}(\xi)$ is done by using (1.8) and (5.6) of [31]. The resulting analytical expression is

$$
\begin{aligned}
& P_{s}(\xi)=\frac{1}{2}-\frac{1}{N 2^{N}} \sum_{q=1}^{2^{N-2}} \sum_{k=0}^{N-1} \\
& \left\{f_{1} \sqrt{\frac{\bar{\gamma}_{k} C_{\beta(k) \beta(k)}\left(\Re[S(0, k)]+z_{q}^{k}\right)^{2}}{1+\bar{\gamma}_{k}\left(C_{\beta(k) \beta(k)}\left(\Re[S(0, k)]+z_{q}^{k}\right)^{2}+a_{q}^{k}\right)}}\right. \\
& \left.+f_{2} \sqrt{\frac{\bar{\gamma}_{k} C_{\beta(k) \beta(k)}\left(\Re[S(0, k)]-z_{q}^{k}\right)^{2}}{1+\bar{\gamma}_{k}\left(C_{\beta(k) \beta(k)}\left(\Re[S(0, k)]-z_{q}^{k}\right)^{2}+a_{q}^{k}\right)}}\right\},
\end{aligned}
$$

where $f_{1}=\operatorname{sgn}\left(\Re[S(0, k)]+z_{q}^{k}\right)$ and $f_{2}=\operatorname{sgn}(\Re[S(0, k)]-$ $\left.z_{q}^{k}\right)$, being $\operatorname{sgn}(\cdot)$ the signum function. By substituting $\alpha=\pi / 2$ in $S(l, k)$ and $C_{\beta(k) \beta(k)}=2 \sigma^{2}$, we get the final expression of SEP for BPSK OFDM system based on DFT given by (13) of [25]. This is conform to the fact that OFDM system based on DFrFT is a generalization of conventional OFDM system.

\section{B. $Q P S K$}

For QPSK modulation the symbols are drawn from the set $\{ \pm 1 \pm j\}$. Without loosing generality, as done in Section III-A, we make the assumption that the symbol transmitted on the $k$ th sub-carrier is $X(k)=1+j$. By following [28] we can write the conditional probability of correct decision as

$$
\begin{aligned}
P_{c} & \left(\beta^{*}(k) Y(k) \in D_{1} \mid X(k)\right. \\
& \left.=1+j, \Re\left[\beta^{*}(k) R(k)\right], \Im\left[\beta^{*}(k) R(k)\right]\right) \\
& =Q\left(-\frac{\Re\left[\beta^{*}(k) R(k)\right]}{\sigma_{\beta}}\right) Q\left(-\frac{\Im\left[\beta^{*}(k) R(k)\right]}{\sigma_{\beta}}\right),
\end{aligned}
$$

where $\Im[\cdot]$ denotes the imaginary part. The probability of correct decision is obtained by averaging (43) over the joint two-dimensional (2D) PDF defined by the real and imaginary parts of $\beta^{*}(k) R(k) \mid X(k)=1+j$, which is given by IFT of the $\mathrm{CHF}$ as

$$
\begin{aligned}
& \varphi_{\Re\left[\beta^{*}(k) R(k) \mid \beta(k), \boldsymbol{\beta}_{k}\right], \Im\left[\beta^{*}(k) R(k) \mid \beta(k), \boldsymbol{\beta}_{k}\right]}\left(\omega_{I}, \omega_{Q}\right) \\
& =e^{j|\beta(k)|^{2}\left(\omega_{I}(\Re[S(0, k)]-\Im[S(0, k)])+\omega_{Q}(\Im[S(0, k)]+\Re[S(0, k)])\right)} \\
& \prod_{\substack{q=0 \\
q \neq k}}^{N-1} \cos \left(\omega_{I} \Re\left[S(q, k) \beta^{*}(k) \beta(q)\right]+\omega_{Q} \Im\left[S(q, k) \beta^{*}(k) \beta(q)\right]\right) \\
& \cos \left(\omega_{I} \Im\left[S(q, k) \beta^{*}(k) \beta(q)\right]-\omega_{Q} \Re\left[S(q, k) \beta^{*}(k) \beta(q)\right]\right) .
\end{aligned}
$$

By following [28], the resulting 2D conditional PDF is

$$
\begin{aligned}
& p\left(\Re\left[\beta^{*}(k) R(k)\right], \Im\left[\beta^{*}(k) R(k)\right] \mid \beta(k), \boldsymbol{\beta}_{k}\right)=\frac{1}{2^{2 N-2}} \sum_{q=0}^{2^{N-2}-1} \\
& \sum_{n=0}^{2^{N-2}-1} \sum_{m=1}^{4} \delta\left[\Re\left(\beta^{*}(k) R(k)\right)-|\beta(k)|^{2}\left(D_{A}+\varphi_{q, n}[1, m]\right)\right] \\
& \delta\left[\Im\left(\beta^{*}(k) R(k)\right)-|\beta(k)|^{2}\left(D_{B}+\varphi_{q, n}[2, m]\right)\right],
\end{aligned}
$$

where $D_{A}=\Re[S(0, k)]-\Im[S(0, k)], D_{B}=\Re[S(0, k)]+\Im$ $[S(0, k)]$ and $\varphi_{q, n}[r, c]$ is the entry $(r, c)$ of the $2 \times 4$ matrix $G$ $=\left[\left(\boldsymbol{g}_{A}+\boldsymbol{g}_{B}\right)\left(-\boldsymbol{g}_{A}-\boldsymbol{g}_{B}\right)\left(\boldsymbol{g}_{A}-\boldsymbol{g}_{B}\right)\left(-\boldsymbol{g}_{A}+\boldsymbol{g}_{B}\right)\right]$ with

$$
\begin{aligned}
& \boldsymbol{g}_{A}=\left[\Re\left[\beta^{*}(k) \boldsymbol{e}_{q}^{T} \boldsymbol{\Lambda} \boldsymbol{\beta}_{k}\right] \quad \Im\left[\beta^{*}(k) \boldsymbol{e}_{n}^{T} \boldsymbol{\Lambda} \boldsymbol{\beta}_{k}\right]\right]^{T}, \\
& \boldsymbol{g}_{B}=\left[\Im\left[\beta^{*}(k) \boldsymbol{e}_{q}^{T} \boldsymbol{\Lambda} \boldsymbol{\beta}_{k}\right]-\Re\left[\beta^{*}(k) \boldsymbol{e}_{n}^{T} \boldsymbol{\Lambda} \boldsymbol{\beta}_{k}\right]\right]^{T} .
\end{aligned}
$$

The expression of the conditional probability of correct decision $P_{c}\left(\beta^{*}(k) R(k) \in D_{1} \mid \beta(k), \boldsymbol{\beta}_{k}\right)$ is therefore obtained by averaging (43) over the 2D PDF in (45). The conditional SEP on the $k$ th sub-carrier is obtained by subtracting the corresponding conditional probability of correct decision from 1 as

$$
\begin{aligned}
& P_{s}\left(\xi_{k} \mid \beta(k), \boldsymbol{\beta}_{k}\right)=1-P_{c}\left(\beta^{*}(k) R(k) \in D_{1} \mid \beta(k), \boldsymbol{\beta}_{k}\right) \\
& =1-\frac{1}{N 2^{2 N-2}} \sum_{q=0}^{2^{N-2}-1} \sum_{n=0}^{2^{N-2}-1} \sum_{m=1}^{4}
\end{aligned}
$$




$$
\begin{aligned}
& Q\left(-\frac{|\beta(k)|^{2}\left(D_{A}+\varphi_{q, n}[1, m]\right)}{\sigma_{\beta}}\right) \\
& Q\left(-\frac{|\beta(k)|^{2}\left(D_{B}+\varphi_{q, n}[2, m]\right)}{\sigma_{\beta}}\right) .
\end{aligned}
$$

As seen in Section III-A, computing $P_{s}\left(\xi_{k} \mid \beta(k), \boldsymbol{\beta}_{k}\right)$ requires the solution of a multi-dimensional integral. Therefore, first we need to average $P_{s}\left(\xi_{k} \mid \beta(k), \boldsymbol{\beta}_{k}\right)$ over $p_{\boldsymbol{\beta}_{k} \mid \beta(k)}\left(\boldsymbol{\beta}_{k} \mid \beta(k)\right)$, then the expression of $P_{s}\left(\xi_{k} \mid \beta(k)\right)$ is obtained by using the mean $|\beta(k)|^{2} \vartheta_{q, n}[i, m]$ and variance $|\beta(k)|^{2} \nu_{q, n}[m] / 2$ of the conditional Gaussian random variable $\varphi_{q, n}[i, m] \mid \beta(k)$ as

$$
\begin{gathered}
P_{s}\left(\xi_{k} \mid \beta(k)\right)=1-\frac{1}{N 2^{2 N-2}} \sum_{q=0}^{2^{N-2}-1} \sum_{n=0}^{2^{N-2}-1} \sum_{m=1}^{4} \\
Q\left(-\frac{|\beta(k)|\left(D_{A}+\vartheta_{q, n}[1, m]\right)}{\sigma \sqrt{1+\frac{\nu_{q, n}[m]}{2 \sigma^{2}}}}\right) \\
Q\left(-\frac{|\beta(k)|\left(D_{B}+\vartheta_{q, n}[2, m]\right)}{\sigma \sqrt{1+\frac{\nu_{q, n}[m]}{2 \sigma^{2}}}}\right),
\end{gathered}
$$

where

- $\vartheta_{q, n}[i, m]$ is the entry $(i, m)$ of the $2 \times 4$ matrix $\boldsymbol{W}=$ $\left[\left(\boldsymbol{w}_{A}+\boldsymbol{w}_{B}\right)\left(-\boldsymbol{w}_{A}-\boldsymbol{w}_{B}\right)\left(\boldsymbol{w}_{A}-\boldsymbol{w}_{B}\right)\left(-\boldsymbol{w}_{A}+\boldsymbol{w}_{B}\right)\right]$ with

$$
\begin{aligned}
& \boldsymbol{w}_{A}=C_{\beta(k) \beta(k)}^{-1}\left[\Re\left[\boldsymbol{e}_{q}^{T} \boldsymbol{\Lambda} \boldsymbol{C}_{\boldsymbol{\beta}_{k} \beta(k)}\right] \Im\left[\boldsymbol{e}_{q}^{T} \boldsymbol{\Lambda} \boldsymbol{C}_{\boldsymbol{\beta}_{k} \beta(k)}\right]\right]^{T}, \\
& \boldsymbol{w}_{B}=C_{\beta(k) \beta(k)}^{-1}\left[\Im\left[\boldsymbol{e}_{n}^{T} \boldsymbol{\Lambda} \boldsymbol{C}_{\boldsymbol{\beta}_{k} \beta(k)}\right]-\Re\left[\boldsymbol{e}_{n}^{T} \boldsymbol{\Lambda} \boldsymbol{C}_{\boldsymbol{\beta}_{k} \beta(k)}\right)\right]^{T},
\end{aligned}
$$

being $\boldsymbol{C}_{\boldsymbol{\beta}_{k} \mid \beta(k)}$ the channel auto-covariance matrix given in [25];

- $\nu_{q, n}[m]$ is the entry $m$ of the $1 \times 4$ vector $\boldsymbol{\nu}=\left[\begin{array}{ll}\nu_{1} & \nu_{2}\end{array}\right.$ $\left.\nu_{3} \nu_{4}\right]$, where $\nu_{i}=\boldsymbol{\zeta}_{i}^{T} \boldsymbol{\Lambda} \boldsymbol{C}_{\boldsymbol{\beta}_{k} \beta(k)} \boldsymbol{\Lambda} \boldsymbol{\zeta}_{i}$ and $\boldsymbol{\zeta}_{1}=\boldsymbol{e}_{q}+\boldsymbol{e}_{n}$, $\boldsymbol{\zeta}_{2}=-\boldsymbol{e}_{q}-\boldsymbol{e}_{n}, \boldsymbol{\zeta}_{3}=\boldsymbol{e}_{q}-\boldsymbol{e}_{n}, \boldsymbol{\zeta}_{4}=-\boldsymbol{e}_{q}+\boldsymbol{e}_{n}$.

We convert the multiplication of the two $Q$-functions in (47) into the sum of two $Q$-functions, which is given in equation (4.8) of [31]. Then, we apply the polar form definition of the $Q$-function in (47) and, as done in Section III-A, introduce $\gamma_{k}$ to express the instantaneous SNR on $k$ th sub-carrier. Following (39), the resulting SEP is (48), shown at the bottom of this page.
The equation can be further simplified by using [31, eq. (1.8)] and, by using the MGF of Rayleigh fading given in (41), the resulting expression can be solved using (5.102) of [31]. After some mathematical passages, the SEP expression is given by

$$
\begin{aligned}
& P_{s}(\xi)=\frac{3}{4}-\frac{1}{N 2^{2 N-1} \sum_{k=0}^{N-1} \sum_{q=0}^{2^{N-2}-1} \sum_{n=0}^{2^{N-2}-1} \sum_{m=1}^{4}} \\
& \sqrt{\frac{C_{\beta(k) \beta(k)} \bar{\gamma}_{k} \Psi_{q, n}^{2}[1, m]}{2+\bar{\gamma}_{k} \nu_{q, n}[m]+C_{\beta(k) \beta(k)} \bar{\gamma}_{k} \Psi_{q, n}^{2}[1, m]}} \\
& \left(\frac{1}{2}+\frac{1}{\pi} \operatorname{atan}\left\{\sqrt{\frac{C_{\beta(k) \beta(k)} \bar{\gamma}_{k} \Psi_{q, n}^{2}[2, m]}{2+\bar{\gamma}_{k} \nu_{q, n}[m]+C_{\beta(k) \beta(k)} \bar{\gamma}_{k} \Psi_{q, n}^{2}[1, m]}}\right\}\right) \\
& +\sqrt{\frac{C_{\beta(k) \beta(k) \Psi_{q, n}^{2}[2, m]} \bar{\gamma}_{k}}{2+\bar{\gamma}_{k} \nu_{q, n}[m]+C_{\beta(k) \beta(k)} \bar{\gamma}_{k} \Psi_{q, n}^{2}[2, m]}} \\
& \left(\frac{1}{2}+\frac{1}{\pi} \operatorname{atan}\left\{\sqrt{\frac{C_{\beta(k) \beta(k)} \bar{\gamma}_{k} \Psi_{q, n}^{2}[1, m]}{2+\bar{\gamma}_{k} \nu_{q, n}[m]+C_{\beta(k) \beta(k)} \bar{\gamma}_{k} \Psi_{q, n}^{2}[2, m]}}\right\}\right),
\end{aligned}
$$

where $\Psi_{q, n}[i, m]$ is the entry $(i, m)$ of the $2 \times 4$ matrix $\boldsymbol{\Phi}=\boldsymbol{D} \otimes \mathbf{1}_{1 \times 4}+\boldsymbol{W}$, in which $\otimes$ is the Kronecker product, $\mathbf{1}_{1 \times 4}$ is a $1 \times 4$ vectors of $1 \mathrm{~s}$, and $\boldsymbol{D}=\left[D_{A} D_{B}\right]^{\mathrm{T}}$. Similarly, substituting $\alpha=\pi / 2$ in $S(l, k)$ and $C_{\beta(k) \beta(k)}=2 \sigma^{2}$ in (49) we get the SEP expression of QPSK for conventional OFDM system given by (16) of [14].

\section{16-QAM}

For 16-QAM symbols are drawn from the set $\{ \pm 1 \pm j, \pm 1 \pm$ $3 j, \pm 3 \pm j, \pm 3 \pm 3 j\}$. We assume that symbol $X(k)$ transmitted on the $k$ th sub-carrier takes a value in the subset $\{1+j, 1+3 j, 3+j, 3+3 j\}$, which corresponds to points in the first quadrant $D_{1}[33]$.

First we consider that the transmitted symbol is $X(k)=1+j$ and evaluates the probability that the equalized received signal lies in the region $D_{s 1}=\{a+b j \mid 2 \geq a \geq 0,2 \geq b \geq 0\}$ of the complex plane. Thus, the conditional probability of making a correct decision given $X(k)=1+j$ is

$$
\begin{aligned}
& P_{c, 1}\left(\beta^{*}(k) Y(k) \in D_{s 1} \mid X(k)\right. \\
& \left.=1+j, \Re\left[\beta^{*}(k) R(k)\right], \Im\left[\beta^{*}(k) R(k)\right]\right)
\end{aligned}
$$

$$
\begin{aligned}
& P_{s}(\xi)=1-\frac{1}{2 \pi N 2^{N-2}} \sum_{k=0}^{N-1} \sum_{q=0}^{2^{N-2}} \sum_{n=0}^{2^{N-2}-1} \sum_{m=1}^{4}\{\int_{0}^{\frac{\pi}{2}-\tan ^{-1}\left(\frac{D_{B}+\vartheta q, n}{\left.D_{A}+\vartheta, m\right]}\right)} \underbrace{\int_{0}^{\infty} \exp \left(-\frac{\gamma_{k}\left(D_{A}+\vartheta_{q, n}[1, m]\right)^{2}}{\left(1+\frac{\nu_{q, n}[m]}{2 \sigma^{2}}\right) \sin ^{2} \theta}\right) p_{\gamma_{k}}\left(\gamma_{k}\right) d \gamma_{k}}_{M_{\gamma_{k}}\left(s_{1}\right)} \\
& +\int_{0}^{\tan ^{-1}\left(\frac{D_{B}+\vartheta q, n[2, m]}{D_{A}+\vartheta q, n[1, m]}\right)} \underbrace{\int_{0}^{\infty} \exp \left(-\frac{\gamma_{k}\left(D_{B}+\vartheta_{q, n}[2, m]\right)^{2}}{\left(1+\frac{\nu_{q, n}[m]}{2 \sigma^{2}}\right) \sin ^{2} \theta}\right) p_{\gamma_{k}}\left(\gamma_{k}\right) d \gamma_{k}}_{M_{\gamma_{k}}\left(s_{2}\right)}\}
\end{aligned}
$$




$$
\begin{aligned}
& =\int_{0}^{2} \frac{1}{\sqrt{2 \pi} \sigma} e^{-\frac{\left(x-\Re\left[\beta^{*}(k) R(k)\right]\right)^{2}}{2 \sigma^{2}}} d x \int_{0}^{2} \frac{1}{\sqrt{2 \pi} \sigma} e^{-\frac{\left(y-\Im\left[\beta^{*}(k) R(k)\right]\right)^{2}}{2 \sigma^{2}}} d y \\
& =\left[Q\left(\frac{-\Re\left[\beta^{*}(k) R(k)\right]}{\sigma_{\beta}}\right)-Q\left(\frac{2-\Re\left[\beta^{*}(k) R(k)\right]}{\sigma_{\beta}}\right)\right] \\
& {\left[Q\left(\frac{-\Im\left[\beta^{*}(k) R(k)\right]}{\sigma_{\beta}}\right)-Q\left(\frac{2-\Im\left[\beta^{*}(k) R(k)\right]}{\sigma_{\beta}}\right)\right]}
\end{aligned}
$$

The probability of correct decision is obtained by averaging (50) over the joint 2D PDF of $\beta^{*}(k) R(k)$ as

$$
\begin{aligned}
& P_{c, 1 \text { avg }}\left(\beta^{*}(k) Y(k) \in D_{s 1} \mid X(k)=1+j\right) \\
&= \int_{-\infty}^{\infty} \int_{-\infty}^{\infty} P_{c, 1}\left(\beta^{*}(k) Y(k) \in D_{s 1} \mid X(k)\right. \\
&=\left.1+j, \Re\left[\beta^{*}(k) R(k)\right], \Im\left[\beta^{*}(k) R(k)\right]\right) \\
& p\left(\Re\left[\beta^{*}(k) R(k)\right], \Im\left[\beta^{*}(k) R(k)\right] \mid X(k)\right. \\
&=1+j) d \Re\left[\beta^{*}(k) R(k)\right] d \Im\left[\beta^{*}(k) R(k)\right],
\end{aligned}
$$

where the expression of $p\left(\Re\left[\beta^{*}(k) R(k)\right], \Im\left[\beta^{*}(k) R(k)\right] \mid X(k)\right.$ $=1+j$ ) is given in [34]. Following [28], after substituting the value of the equalized signal $\beta^{*}(k) R(k)$ and after some simplifications, the 2D-CHF can be written as

$\varphi_{\Re\left[\beta^{*}(k) R(k) \mid \beta(k), \boldsymbol{\beta}_{k}\right], \Im\left[\beta^{*}(k) R(k) \mid \beta(k), \boldsymbol{\beta}_{k}\right]}\left(\omega_{I}, \omega_{Q}\right)$

$=E\left[e^{j|\beta(k)|^{2}\left(\Omega^{T} \boldsymbol{S}_{\boldsymbol{a}}(0, k) \Re[X(k)]-\Omega^{T} \boldsymbol{S}_{\boldsymbol{b}}(0, k) \Im[X(k)]\right)}\right]$

$\prod_{q=0}^{N-1} E\left[e^{j\left(\beta^{*}(k) \beta(q) \Omega^{T} \boldsymbol{S}_{\boldsymbol{a}}(q, k) \Re[X(q)]-\beta^{*}(k) \beta(q) \Omega^{T} \boldsymbol{S}_{\boldsymbol{b}}(q, k) \Im[X(q)]\right)}\right]$, $q \neq k$

where $\Omega^{T}=\left[\omega_{I} \omega_{Q}\right], \boldsymbol{S}_{\boldsymbol{a}}(q, k)=[\Re[S(q, k)] \Im[S(q, k)]]^{T}$ and $\boldsymbol{S}_{\boldsymbol{b}}(q, k)=[\Im[S(q, k)]-\Re[S(q, k)]]^{T}$. By substituting the value of $X(k)=1+j$ in (52) and by expanding in terms of real and imaginary parts we get

$\varphi_{\Re\left[\beta^{*}(k) R(k) \mid \beta(k), \boldsymbol{\beta}_{k}\right], \Im\left[\beta^{*}(k) R(k) \mid \beta(k), \boldsymbol{\beta}_{k}\right]}\left(\omega_{I}, \omega_{Q}\right)$

$=E\left[e^{\left[j|\beta(k)|^{2}\left\{\omega_{I}\left(\Re[S(0, k)]-\Im[S(0, k)]+\omega_{Q}(\Im[S(0, k)]+\Re[S(0, k)])\right\}\right]\right.}\right]$ $\prod_{\substack{q=0 \\ q \neq k}}^{N-1} \cos \left(\omega_{I} \Re\left[S(q, k) \beta^{*}(k) \beta(q)\right]+\omega_{Q} \Im\left[S(q, k) \beta^{*}(k) \beta(q)\right]\right)$

$\cos \left(2 \omega_{I} \Re\left[S(q, k) \beta^{*}(k) \beta(q)\right]+2 \omega_{Q} \Im\left[S(q, k) \beta^{*}(k) \beta(q)\right]\right)$

$\cos \left(\omega_{I} \Im\left[S(q, k) \beta^{*}(k) \beta(q)\right]-\omega_{Q} \Re\left[S(q, k) \beta^{*}(k) \beta(q)\right]\right)$

$\cos \left(2 \omega_{I} \Im\left[S(q, k) \beta^{*}(k) \beta(q)\right]-2 \omega_{Q} \Re\left[S(q, k) \beta^{*}(k) \beta(q)\right]\right)$.

The resulting PDF, obtained by computing the IFT of (53), is

$$
\begin{aligned}
p(\Re[ & {\left.\left[\beta^{*}(k) R(k)\right], \Im\left[\beta^{*}(k) R(k)\right]\right) } \\
= & \frac{1}{2^{4 N-2}} \sum_{q=0}^{2^{N-2}-1} \sum_{l=0}^{2^{N-2}-1} \sum_{m=0}^{2^{N-2}-1} \sum_{n=0}^{2^{N-2}-1} \sum_{f=1}^{16} \delta\left[\Re\left[\beta^{*}(k) \Re(k)\right]\right. \\
& \left.-\left(|\beta(k)|^{2}\left(D_{A}+\boldsymbol{\zeta}_{q, l, m, n}[1, f]\right)\right)\right]
\end{aligned}
$$$$
\delta\left[\Im\left[\beta^{*}(k) R(k)\right]-\left(|\beta(k)|^{2}\left(D_{B}+\boldsymbol{\zeta}_{q, l, m, n}[2, f]\right)\right)\right],
$$

where $\zeta_{q, l, m, n}[p, q]$ is the entry of the $2 \times 16$ matrix of

$$
\begin{aligned}
\boldsymbol{G}= & {\left[\left(\boldsymbol{g}_{A}+\boldsymbol{g}_{B}+\boldsymbol{g}_{C}+\boldsymbol{g}_{D}\right)\left(\boldsymbol{g}_{A}+\boldsymbol{g}_{B}+\boldsymbol{g}_{C}-\boldsymbol{g}_{D}\right)\right.} \\
& \left(\boldsymbol{g}_{A}+\boldsymbol{g}_{B}-\boldsymbol{g}_{C}+\boldsymbol{g}_{D}\right)\left(\boldsymbol{g}_{A}+\boldsymbol{g}_{B}-\boldsymbol{g}_{C}-\boldsymbol{g}_{D}\right) \\
& \left(-\boldsymbol{g}_{A}-\boldsymbol{g}_{B}+\boldsymbol{g}_{C}+\boldsymbol{g}_{D}\right)\left(-\boldsymbol{g}_{A}-\boldsymbol{g}_{B}+\boldsymbol{g}_{C}-\boldsymbol{g}_{D}\right) \\
& \left(-\boldsymbol{g}_{A}-\boldsymbol{g}_{B}-\boldsymbol{g}_{C}+\boldsymbol{g}_{D}\right)\left(-\boldsymbol{g}_{A}-\boldsymbol{g}_{B}-\boldsymbol{g}_{C}-\boldsymbol{g}_{D}\right) \\
& \left(\boldsymbol{g}_{A}-\boldsymbol{g}_{B}+\boldsymbol{g}_{C}+\boldsymbol{g}_{D}\right)\left(\boldsymbol{g}_{A}-\boldsymbol{g}_{B}+\boldsymbol{g}_{C}-\boldsymbol{g}_{D}\right) \\
& \left(\boldsymbol{g}_{A}-\boldsymbol{g}_{B}-\boldsymbol{g}_{C}+\boldsymbol{g}_{D}\right)\left(\boldsymbol{g}_{A}-\boldsymbol{g}_{B}-\boldsymbol{g}_{C}-\boldsymbol{g}_{D}\right) \\
& \left(-\boldsymbol{g}_{A}+\boldsymbol{g}_{B}+\boldsymbol{g}_{C}+\boldsymbol{g}_{D}\right)\left(-\boldsymbol{g}_{A}+\boldsymbol{g}_{B}+\boldsymbol{g}_{C}-\boldsymbol{g}_{D}\right) \\
& \left.\left(-\boldsymbol{g}_{A}+\boldsymbol{g}_{B}-\boldsymbol{g}_{C}+\boldsymbol{g}_{D}\right)\left(-\boldsymbol{g}_{A}+\boldsymbol{g}_{B}-\boldsymbol{g}_{C}-\boldsymbol{g}_{D}\right)\right]
\end{aligned}
$$

with

$$
\begin{aligned}
& \boldsymbol{g}_{A}=\left[\Re\left[\beta^{*}(k) \boldsymbol{\Lambda} \boldsymbol{\beta}_{k} e_{q}^{T}\right] \quad \Im\left[\beta^{*}(k) \boldsymbol{\Lambda} \boldsymbol{\beta}_{k} e_{q}^{T}\right]\right]^{T}, \\
& \boldsymbol{g}_{B}=\left[2 \Re\left[\beta^{*}(k) \boldsymbol{\Lambda} \boldsymbol{\beta}_{k} e_{l}^{T}\right] \quad 2 \Im\left[\beta^{*}(k) \boldsymbol{\Lambda} \boldsymbol{\beta}_{k} e_{l}^{T}\right]\right]^{T} \text {, }
\end{aligned}
$$

$P_{c, 1 \text { avg }}\left(\beta^{*}(k) Y(k) \in D_{s 1} \mid \beta(k), \boldsymbol{\beta}_{k}\right)=\frac{1}{2^{4 N-2}} \sum_{q=0}^{2^{N-2}} \sum_{l=0}^{-1} \sum_{m=0}^{2^{N-2}-1} \sum_{n=0}^{2^{N-2}} \sum_{f=1}^{16}$

$\mathrm{Q}\left(\frac{-\left(|\beta(k)|^{2} D_{A}+\boldsymbol{\zeta}_{q, l, m, n}[1, f]\right)}{\sigma_{\beta}}\right) \mathrm{Q}\left(\frac{-\left(|\beta(k)|^{2} D_{B}+\boldsymbol{\zeta}_{q, l, m, n}[2, f]\right)}{\sigma_{\beta}}\right)-\mathrm{Q}\left(\frac{-\left(|\beta(k)|^{2} D_{A}+\boldsymbol{\zeta}_{q, l, m, n}[1, f]\right)}{\sigma_{\beta}}\right)$

$\mathrm{Q}\left(\frac{2-\left(|\beta(k)|^{2} D_{B}+\boldsymbol{\zeta}_{q, l, m, n}[2, f]\right)}{\sigma_{\beta}}\right)-\mathrm{Q}\left(\frac{2-\left(|\beta(k)|^{2} D_{A}+\boldsymbol{\zeta}_{q, l, m, n}[1, f]\right)}{\sigma_{\beta}}\right) \mathrm{Q}\left(\frac{-\left(|\beta(k)|^{2} D_{B}+\boldsymbol{\zeta}_{q, l, m, n}[2, f]\right)}{\sigma_{\beta}}\right)$

$+\mathrm{Q}\left(\frac{2-\left(|\beta(k)|^{2} D_{A}+\boldsymbol{\zeta}_{q, l, m, n}[1, f]\right)}{\sigma_{\beta}}\right) \mathrm{Q}\left(\frac{2-\left(|\beta(k)|^{2} D_{B}+\boldsymbol{\zeta}_{q, l, m, n}[2, f]\right)}{\sigma_{\beta}}\right)$ 


$$
\begin{aligned}
& \boldsymbol{g}_{C}=\left[\Im\left[\beta^{*}(k) \boldsymbol{\Lambda} \boldsymbol{\beta}_{k} e_{m}^{T}\right]-\Re\left[\beta^{*}(k) \boldsymbol{\Lambda} \boldsymbol{\beta}_{k} e_{m}^{T}\right]\right]^{T}, \\
& \boldsymbol{g}_{D}=\left[2 \Im\left[\beta^{*}(k) \boldsymbol{\Lambda} \boldsymbol{\beta}_{k} e_{n}^{T}\right]-2 \Re\left[\beta^{*}(k) \boldsymbol{\Lambda} \boldsymbol{\beta}_{k} e_{n}^{T}\right]\right]^{T} .
\end{aligned}
$$

The PDF given in (54) is a function of $\boldsymbol{\beta}$ and, therefore, by substituting in (51), we get (55), shown at the bottom of the previous page. It is worth observing that in the above equation the dependence on $\left(\beta(k), \boldsymbol{\beta}_{k}\right)$ is implicit in $\boldsymbol{\zeta}_{q, l, m, n}[1, f]$ and $\boldsymbol{\zeta}_{q, l, m, n}[2, f]$.

When symbol $X(k)=1+3 j$ is considered, a correct decision is taken when the equalized received signal lies in the region $D_{s 2}=\{a+b j \mid 2 \geq a \geq 0, b \geq 2\}$. By repeating the same considerations done for symbol $X(k)=1+j$, it is possible to derive the expression of the probability of correct decision conditioned to the transmission of symbol $X(k)=1+3 j$ as

$$
\begin{aligned}
P_{c, 2 a v g}\left(\beta^{*}(k) Y(k) \in D_{s 2} \mid \beta(k), \boldsymbol{\beta}_{k}\right) & \frac{1}{2^{4 N-2}} \sum_{q=0}^{2^{N-2}-1} \sum_{l=0}^{2^{N-2}-1} \sum_{m=0}^{2^{N-2}-1} \sum_{n=0}^{2^{N-2}-1} \sum_{f=1}^{16} \\
& \mathrm{Q}\left(\frac{-\left(|\beta(k)|^{2} D_{A}+\boldsymbol{\zeta}_{q, l, m, n}[1, f]\right)}{\sigma_{\beta}}\right) \\
& \mathrm{Q}\left(\frac{2-\left(|\beta(k)|^{2} D_{B}+\boldsymbol{\zeta}_{q, l, m, n}[2, f]\right)}{\sigma_{\beta}}\right) \\
& -\mathrm{Q}\left(\frac{2-\left(|\beta(k)|^{2} D_{A}+\boldsymbol{\zeta}_{q, l, m, n}[1, f]\right)}{\sigma_{\beta}}\right) \\
& \mathrm{Q}\left(\frac{2-\left(|\beta(k)|^{2} D_{B}+\boldsymbol{\zeta}_{q, l, m, n}[2, f]\right)}{\sigma_{\beta}}\right) .
\end{aligned}
$$

Similarly, for transmitted symbol $X(k)=3+j$, the correct decision lies in the region $D_{s 3}=\{a+b j \mid a \geq 2,2 \geq b \geq 0\}$

$$
\begin{aligned}
P_{c, 3 a v g}\left(\beta^{*}(k) Y(k) \in D_{s 3} \mid \beta(k), \boldsymbol{\beta}_{k}\right) & \frac{1}{2^{4 N-2}} \sum_{q=0}^{2^{N-2}-12^{N-2}-12^{N-2}-12^{N-2}-1} \sum_{m=0}^{16} \sum_{n=0}^{16} \sum_{f=1} \\
& \mathrm{Q}\left(\frac{2-\left(|\beta(k)|^{2} D_{A}+\boldsymbol{\zeta}_{q, l, m, n}[1, f]\right)}{\sigma_{\beta}}\right) \\
& \mathrm{Q}\left(\frac{-\left(|\beta(k)|^{2} D_{B}+\boldsymbol{\zeta}_{q, l, m, n}[2, f]\right)}{\sigma_{\beta}}\right) \\
- & \mathrm{Q}\left(\frac{2-\left(|\beta(k)|^{2} D_{A}+\boldsymbol{\zeta}_{q, l, m, n}[1, f]\right)}{\sigma_{\beta}}\right) . \\
& \mathrm{Q}\left(\frac{2-\left(|\beta(k)|^{2} D_{B}+\boldsymbol{\zeta}_{q, l, m, n}[2, f]\right)}{\sigma_{\beta}}\right) .
\end{aligned}
$$

Finally, for transmitted symbol $X(k)=3+3 j$, the correct decision lies in the region $D_{s 4}=\{a+b j \mid a \geq 2, b \geq 2\}$

$$
\begin{aligned}
P_{c, 4 a v g}\left(\beta^{*}(k) Y(k) \in D_{s 4} \mid \beta(k), \boldsymbol{\beta}_{k}\right) & \frac{1}{2^{4 N-2}} \sum_{q=0}^{2^{N-2}-1} \sum_{l=0}^{2^{N-2}-1} \sum_{m=0}^{2^{N-2}-1} \sum_{n=0}^{2^{N-2}-1} \sum_{f=1}^{16} \\
& \mathrm{Q}\left(\frac{2-\left(|\beta(k)|^{2} D_{A}+\boldsymbol{\zeta}_{q, l, m, n}[1, f]\right)}{\sigma_{\beta}}\right) \\
& \mathrm{Q}\left(\frac{2-\left(|\beta(k)|^{2} D_{B}+\boldsymbol{\zeta}_{q, l, m, n}[2, f]\right)}{\sigma_{\beta}}\right) .
\end{aligned}
$$

By taking into account the symmetry of the 16-QAM modulation, the average probability of correct decision is

$P_{c}\left(\xi_{k} \mid \beta(k), \boldsymbol{\beta}_{k}\right)=\frac{1}{4} \sum_{z=1}^{4} P_{c, z a v g}\left(\beta^{*}(k) Y(k) \in D_{s z} \mid \beta(k), \boldsymbol{\beta}_{k}\right)$.

The analytical expression of the SEP given $\left(\beta(k), \boldsymbol{\beta}_{k}\right)$ is

$$
\begin{aligned}
& P_{s}\left(\xi_{k} \mid \beta(k), \boldsymbol{\beta}_{k}\right) \triangleq P_{s}\left(\beta^{*}(k) Y(k) \notin D_{s 1} \mid \beta(k), \boldsymbol{\beta}_{k}\right) \\
& \quad=1-P_{c}\left(\beta^{*}(k) Y(k) \in D_{s 1} \mid \beta(k), \boldsymbol{\beta}_{k}\right)
\end{aligned}
$$

By following (29), the analytical expression of $P_{s}\left(\xi_{k} \mid \beta(k)\right)$ given in (61), shown at the bottom of the next page, is obtained by averaging over the pdf $p_{\boldsymbol{\beta}_{k} \mid \beta(k)}\left(\boldsymbol{\beta}_{k} \mid \beta(k)\right)$, which is described by using the conditional mean $|\beta(k)|^{2} \boldsymbol{\vartheta}_{q, l, m, n}[i, m] \mid \beta(k)$ and the conditional variance $|\beta(k)|^{2} \boldsymbol{\nu}_{q, l, m, n}[m] / 2 \mid \beta(k)$ of the conditional Gaussian random variable $\boldsymbol{\zeta}_{q, l, m, n}[i, f] \mid \beta(k), i=1,2$, where

- $\boldsymbol{\vartheta}_{q, l, m, n}[i, f]$ is the entry $(i, f)$ of the $2 \times 16$ matrix of $\boldsymbol{W}=$

$$
\begin{aligned}
& {\left[\left(\boldsymbol{w}_{A}+\boldsymbol{w}_{B}+\boldsymbol{w}_{C}+\boldsymbol{w}_{D}\right)\left(\boldsymbol{w}_{A}+\boldsymbol{w}_{B}+\boldsymbol{w}_{C}-\boldsymbol{w}_{D}\right)\right.} \\
& \left(\boldsymbol{w}_{A}+\boldsymbol{w}_{B}-\boldsymbol{w}_{C}+\boldsymbol{w}_{D}\right)\left(\boldsymbol{w}_{A}+\boldsymbol{w}_{B}-\boldsymbol{w}_{C}-\boldsymbol{w}_{D}\right) \\
& \left(-\boldsymbol{w}_{A}-\boldsymbol{w}_{B}+\boldsymbol{w}_{C}+\boldsymbol{w}_{D}\right)\left(-\boldsymbol{w}_{A}-\boldsymbol{w}_{B}+\boldsymbol{g}_{C}-\boldsymbol{w}_{D}\right) \\
& \left(-\boldsymbol{w}_{A}-\boldsymbol{w}_{B}-\boldsymbol{w}_{C}+\boldsymbol{w}_{D}\right)\left(-\boldsymbol{w}_{A}-\boldsymbol{w}_{B}-\boldsymbol{w}_{C}-\boldsymbol{w}_{D}\right) \\
& \left(\boldsymbol{w}_{A}-\boldsymbol{w}_{B}+\boldsymbol{w}_{C}+\boldsymbol{w}_{D}\right)\left(\boldsymbol{w}_{A}-\boldsymbol{w}_{B}+\boldsymbol{w}_{C}-\boldsymbol{w}_{D}\right) \\
& \left(\boldsymbol{w}_{A}-\boldsymbol{w}_{B}-\boldsymbol{w}_{C}+\boldsymbol{w}_{D}\right)\left(\boldsymbol{w}_{A}-\boldsymbol{w}_{B}-\boldsymbol{w}_{C}-\boldsymbol{w}_{D}\right) \\
& \left(-\boldsymbol{w}_{A}+\boldsymbol{w}_{B}+\boldsymbol{w}_{C}+\boldsymbol{w}_{D}\right)\left(-\boldsymbol{w}_{A}+\boldsymbol{w}_{B}+\boldsymbol{w}_{C}-\boldsymbol{w}_{D}\right) \\
& \left.\left(-\boldsymbol{w}_{A}+\boldsymbol{w}_{B}-\boldsymbol{w}_{C}+\boldsymbol{w}_{D}\right)\left(-\boldsymbol{w}_{A}+\boldsymbol{w}_{B}-\boldsymbol{w}_{C}-\boldsymbol{w}_{D}\right)\right]
\end{aligned}
$$

with

$$
\begin{aligned}
\boldsymbol{w}_{A} & =C_{\beta(k) \beta(k)}^{-1}\left[\Re\left[\boldsymbol{e}_{q}^{T} \boldsymbol{\Lambda} \boldsymbol{C}_{\boldsymbol{\beta}_{k} \beta(k)}\right] \Im\left[\boldsymbol{e}_{q}^{T} \boldsymbol{\Lambda} \boldsymbol{C}_{\boldsymbol{\beta}_{k} \beta(k)}\right]\right]^{T} \\
\boldsymbol{w}_{B} & =C_{\beta(k) \beta(k)}^{-1}\left[2 \Re\left[\boldsymbol{e}_{l}^{T} \boldsymbol{\Lambda} \boldsymbol{C}_{\boldsymbol{\beta}_{k} \beta(k)}\right] 2 \Im\left[\boldsymbol{e}_{l}^{T} \boldsymbol{\Lambda} \boldsymbol{C}_{\boldsymbol{\beta}_{k} \beta(k)}\right]\right]^{T} \\
\boldsymbol{w}_{C} & =C_{\beta(k) \beta(k)}^{-1}\left[\Im\left[\boldsymbol{e}_{m}^{T} \boldsymbol{\Lambda} \boldsymbol{C}_{\boldsymbol{\beta}_{k} \beta(k)}\right]-\Re\left[\boldsymbol{e}_{m}^{T} \boldsymbol{\Lambda} \boldsymbol{C}_{\boldsymbol{\beta}_{k} \beta(k)}\right]\right]^{T} \\
\boldsymbol{w}_{D} & =C_{\beta(k) \beta(k)}^{-1}\left[2 \Im\left[\boldsymbol{e}_{n}^{T} \boldsymbol{\Lambda} \boldsymbol{C}_{\boldsymbol{\beta}_{k} \beta(k)}\right]-2 \Re\left[\boldsymbol{e}_{n}^{T} \boldsymbol{\Lambda} \boldsymbol{C}_{\boldsymbol{\beta}_{k} \beta(k)}\right]\right]^{T}
\end{aligned}
$$

being $\boldsymbol{C}_{\boldsymbol{\beta}_{k} \mid \beta(k)}$ the channel auto-covariance matrix given in [25]; 
- $\boldsymbol{\nu}_{q, l, m, n}[f]$ is the $f$ th element of the $1 \times 16$ vector $\boldsymbol{\nu}=$ $\left[\begin{array}{llll}\nu_{1} & \nu_{2} & \cdots & \nu_{16}\end{array}\right]$, where $\nu_{i}=\boldsymbol{\zeta}_{i}^{T} \boldsymbol{\Lambda} \boldsymbol{C}_{\boldsymbol{\beta}_{k} \beta(k)} \boldsymbol{\Lambda} \boldsymbol{\zeta}_{i}$ and $\boldsymbol{\zeta}_{1}=$ $\boldsymbol{e}_{q}+\boldsymbol{e}_{l}+\boldsymbol{e}_{m}+\boldsymbol{e}_{n}, \quad \boldsymbol{\zeta}_{2}=\boldsymbol{e}_{q}+\boldsymbol{e}_{l}+\boldsymbol{e}_{m}-\boldsymbol{e}_{n}, \quad \boldsymbol{\zeta}_{3}=\boldsymbol{e}_{q}$ $+\boldsymbol{e}_{l}-\boldsymbol{e}_{m}+\boldsymbol{e}_{n}, \boldsymbol{\zeta}_{4}=\boldsymbol{e}_{q}+\boldsymbol{e}_{l}-\boldsymbol{e}_{m}-\boldsymbol{e}_{n}, \quad \boldsymbol{\zeta}_{5}=-\boldsymbol{e}_{q}-$ $\boldsymbol{e}_{l}+\boldsymbol{e}_{m}+\boldsymbol{e}_{n}, \quad \boldsymbol{\zeta}_{6}=-\boldsymbol{e}_{q}-\boldsymbol{e}_{l}+\boldsymbol{e}_{m}-\boldsymbol{e}_{n}, \quad \boldsymbol{\zeta}_{7}=-\boldsymbol{e}_{q}-$ $e_{l}-e_{m}+e_{n}, \quad \zeta_{8}=-e_{q}-e_{l}-e_{m}-e_{n}, \quad \zeta_{9}=e_{q}-$ $\boldsymbol{e}_{l}+\boldsymbol{e}_{m}+\boldsymbol{e}_{n}, \quad \boldsymbol{\zeta}_{10}=\boldsymbol{e}_{q}-\boldsymbol{e}_{l}+\boldsymbol{e}_{m}-\boldsymbol{e}_{n}, \quad \boldsymbol{\zeta}_{11}=\boldsymbol{e}_{q}-$ $\boldsymbol{e}_{l}-\boldsymbol{e}_{m}+\boldsymbol{e}_{n}, \quad \boldsymbol{\zeta}_{12}=\boldsymbol{e}_{q}-\boldsymbol{e}_{l}-\boldsymbol{e}_{n}-\boldsymbol{e}_{n}, \quad \boldsymbol{\zeta}_{13}=-\boldsymbol{e}_{q}+$ $\boldsymbol{e}_{l}+\boldsymbol{e}_{m}+\boldsymbol{e}_{n}, \boldsymbol{\zeta}_{14}=-\boldsymbol{e}_{q}+\boldsymbol{e}_{l}+\boldsymbol{e}_{m}+\boldsymbol{e}_{n}, \boldsymbol{\zeta}_{15}=-\boldsymbol{e}_{q}+$ $\boldsymbol{e}_{l}-\boldsymbol{e}_{m}+\boldsymbol{e}_{n}, \boldsymbol{\zeta}_{16}=-\boldsymbol{e}_{q}+\boldsymbol{e}_{l}-\boldsymbol{e}_{m}-\boldsymbol{e}_{n}$.

Following the same analysis developed in Section III-B, the computation of the SEP can be performed by replacing the polar form definition of the $Q$-function in (61) and by converting the multiplication of the two $Q$-functions into the sum of two $Q$ functions given in equation (4.8) of [31]. After that, by using (5.102) of [31], the expression of the resulting $P_{s}(\xi)$ is given in (62), shown at the bottom of the next page. Substituting $\alpha=\pi / 2$ in $S(l, k)$ and $C_{\beta(k) \beta(k)}=2 \sigma^{2}$ in (42) we get the SEP expression of 16-QAM for conventional OFDM system given by (51) of [35].

\section{Extension to MASSIVE MIMO}

The analysis presented so far can be easily extended to the case of massive MIMO channel. To this aim, the channel model given in (5) must be modified to include also the spatial characteristic. In particular, by focusing on the case of a uniform planar array with $M$ antennas at the transmitter and a single antenna at the receiver, in [36] a 3D space-time channel model is proposed by associating a steering vector, defined by azimuthal and the elevation angle of departures (AoDs), to each multi-path component. The resulting multipath channel model is

$$
\mathbf{h}(t)=\sum_{l=0}^{L-1} h_{l}(t) \delta\left(t-\tau_{l} T\right) \mathbf{a}\left(\phi_{l}(t), \theta_{l}(t)\right),
$$

where $\mathbf{a}\left(\phi_{l}(t), \theta_{l}(t)\right)$ is the time-varying steering vector related to the $l$ th path defined as

$$
\begin{aligned}
\mathbf{a}\left(\phi_{l}(t), \theta_{l}(t)\right)= & {\left[1, e^{j 2 \pi \frac{d}{\lambda} \sin \left(\theta_{l}(t)\right)}, \ldots, e^{j 2 \pi \frac{d}{\lambda}\left(M_{2}-1\right) \sin \left(\theta_{l}(t)\right)}\right] } \\
\otimes & \frac{1}{\sqrt{M}}\left[1, e^{j 2 \pi \frac{d}{\lambda} \cos \left(\theta_{l}(t)\right) \sin \left(\phi_{l}(t)\right)},\right. \\
& \left.\ldots, e^{j 2 \pi \frac{d}{\lambda}\left(M_{1}-1\right) \cos \left(\theta_{l}(t)\right) \sin \left(\phi_{l}(t)\right)}\right]^{T}, \quad \text { (64) }
\end{aligned}
$$

being $\otimes$ the Kronecker product, $\phi_{u, l}$ and $\theta_{u, l}$ the azimuthal and elevation AoD, respectively, $M_{1}$ and $M_{2}$ the number of horizontal and vertical antennas, respectively, and $M=M_{1} \times$ $M_{2}$. In general, as observed in [37], angle coherence time is higher compared to classical channel coherence time, which leads to slow variations in time of AoDs compared to those of complex amplitudes $h_{l}(t)$. Therefore, when the same block fading assumption done in Sec. II.C is made, the channel model given in (63) can be written as

$$
\mathbf{h}(t)=\sum_{l=0}^{L-1} h_{l} \delta\left(t-\tau_{l} T\right) \mathbf{a}\left(\phi_{l}, \theta_{l}\right) .
$$

By adopting the classical narrowband channel assumption, the above equation reduces to the same spatial channel vector

$$
\begin{aligned}
& P_{s}\left(\xi_{k} \mid \beta(k)\right)=1-\frac{1}{42^{4 N-2}} \sum_{q=0}^{2^{N-2}-1} \sum_{l=0}^{2^{N-2}-1} \sum_{m=0}^{2^{N-2}} \sum_{n=0}^{2^{N-2}} \sum_{f=1}^{16} \mathrm{Q}\left(\frac{-|\beta(k)|\left(D_{A}+\boldsymbol{\vartheta}_{q, l, m, n}[1, f]\right)}{\sigma \sqrt{1+\frac{\boldsymbol{\nu}_{q, l, m, n}[f]}{2 \sigma^{2}}}}\right) \\
& \times \mathrm{Q}\left(\frac{-|\beta(k)|\left(D_{B}+\boldsymbol{\vartheta}_{q, l, m, n}[2, f]\right)}{\sigma \sqrt{1+\frac{\boldsymbol{\nu}_{q, l, m, n}[f]}{2 \sigma^{2}}}}\right)-\mathrm{Q}\left(\frac{-|\beta(k)|\left(D_{A}+\boldsymbol{\vartheta}_{q, l, m, n}[1, f]\right)}{\sigma \sqrt{1+\frac{\boldsymbol{\nu}_{q, l, m, n}[f]}{2 \sigma^{2}}}}\right) \mathrm{Q}\left(\frac{2-|\beta(k)|\left(D_{B}+\boldsymbol{\vartheta}_{q, l, m, n}[2, f]\right)}{\sigma \sqrt{1+\frac{\boldsymbol{\nu}_{q, l, m, n}[f]}{2 \sigma^{2}}}}\right) \\
& -\mathrm{Q}\left(\frac{2-|\beta(k)|\left(D_{A}+\boldsymbol{\vartheta}_{q, l, m, n}[1, f]\right)}{\sigma \sqrt{1+\frac{\boldsymbol{\nu}_{q, l, m, n}[f]}{2 \sigma^{2}}}}\right) \mathrm{Q}\left(\frac{-|\beta(k)|\left(D_{B}+\boldsymbol{\vartheta}_{q, l, m, n}[2, f]\right)}{\sigma \sqrt{1+\frac{\boldsymbol{\nu}_{q, l, m, n}[f]}{2 \sigma^{2}}}}\right)+\mathrm{Q}\left(\frac{2-|\beta(k)|\left(D_{A}+\boldsymbol{\vartheta}_{q, l, m, n}[1, f]\right)}{\sigma \sqrt{1+\frac{\boldsymbol{\nu}_{q, l, m, n}[f]}{2 \sigma^{2}}}}\right) \\
& \mathrm{Q}\left(\frac{2-|\beta(k)|\left(D_{B}+\boldsymbol{\vartheta}_{q, l, m, n}[2, f]\right)}{\sigma \sqrt{1+\frac{\boldsymbol{\nu}_{q, l, m, n}[f]}{2 \sigma^{2}}}}\right)+-\mathrm{Q}\left(\frac{-|\beta(k)|\left(D_{A}+\boldsymbol{\vartheta}_{q, l, m, n}[1, f]\right)}{\sigma \sqrt{1+\frac{\boldsymbol{\nu}_{q, l, m, n}[f]}{2 \sigma^{2}}}}\right) \mathrm{Q}\left(\frac{2-|\beta(k)|\left(D_{B}+\boldsymbol{\vartheta}_{q, l, m, n}[2, f]\right)}{\sigma \sqrt{1+\frac{\boldsymbol{\nu}_{q, l, m, n}[f]}{2 \sigma^{2}}}}\right) \\
& -\mathrm{Q}\left(\frac{2-|\beta(k)|\left(D_{A}+\boldsymbol{\vartheta}_{q, l, m, n}[1, f]\right)}{\sigma \sqrt{1+\frac{\boldsymbol{\nu}_{q, l, m, n}[f]}{2 \sigma^{2}}}}\right) \mathrm{Q}\left(\frac{2-|\beta(k)|\left(D_{B}+\boldsymbol{\vartheta}_{q, l, m, n}[2, f]\right)}{\sigma \sqrt{1+\frac{\boldsymbol{\nu}_{q, l, m, n}[f]}{2 \sigma^{2}}}}\right)+-\mathrm{Q}\left(\frac{2-|\beta(k)|\left(D_{A}+\boldsymbol{\vartheta}_{q, l, m, n}[1, f]\right)}{\sigma \sqrt{1+\frac{\boldsymbol{\nu}_{q, l, m, n}[f]}{2 \sigma^{2}}}}\right) \\
& \times \mathrm{Q}\left(\frac{-|\beta(k)|\left(D_{B}+\boldsymbol{\vartheta}_{q, l, m, n}[2, f]\right)}{\sigma \sqrt{1+\frac{\boldsymbol{\nu}_{q, l, m, n}[f]}{2 \sigma^{2}}}}\right)-\mathrm{Q}\left(\frac{2-|\beta(k)|\left(D_{A}+\boldsymbol{\vartheta}_{q, l, m, n}[1, f]\right)}{\sigma \sqrt{1+\frac{\boldsymbol{\nu}_{q, l, m, n}[f]}{2 \sigma^{2}}}}\right) \mathrm{Q}\left(\frac{2-|\beta(k)|\left(D_{B}+\boldsymbol{\vartheta}_{q, l, m, n}[2, f]\right)}{\sigma \sqrt{1+\frac{\boldsymbol{\nu}_{q, l, m, n}[f]}{2 \sigma^{2}}}}\right) \\
& +-\mathrm{Q}\left(\frac{2-|\beta(k)|\left(D_{A}+\boldsymbol{\vartheta}_{q, l, m, n}[1, f]\right)}{\sigma \sqrt{1+\frac{\boldsymbol{\nu}_{q, l, m, n}[f]}{2 \sigma^{2}}}}\right) \mathrm{Q}\left(\frac{2-|\beta(k)|\left(D_{B}+\boldsymbol{\vartheta}_{q, l, m, n}[2, f]\right)}{\sigma \sqrt{1+\frac{\boldsymbol{\nu}_{q, l, m, n}[f]}{2 \sigma^{2}}}}\right)
\end{aligned}
$$


defined by [36, eq. (1)] giving rise to

$$
\mathbf{h}=\sum_{l=0}^{L-1} h_{l} \mathbf{a}\left(\phi_{l}, \theta_{l}\right) .
$$

In massive MIMO, the spatial channel model defined in (66) is used by the transmitter to perform beamforming where the resulting channel is modeled as a scalar coefficient. This defines a frequency flat fading channel for which it is straightforward to analyze the performance in the presence of CFO and STO by using the analytical expressions derived in Section III.

Since this paper deals with the analysis of performance in presence of CFO and STO for transmission over a frequency selective channel, the SEP analysis for massive MIMO is left to future work. No simulation results are therefore reported in Section VI.

\section{Optimal DFrFT ANGLE PARAMETER}

From Fig. 2 it is clearly visible that for a given $\Delta \epsilon$ the optimal value of the DFrFT angle parameter $\alpha$ is independent from the modulation scheme. The reason of this independence can be easily explained from (22), which gives the model of the

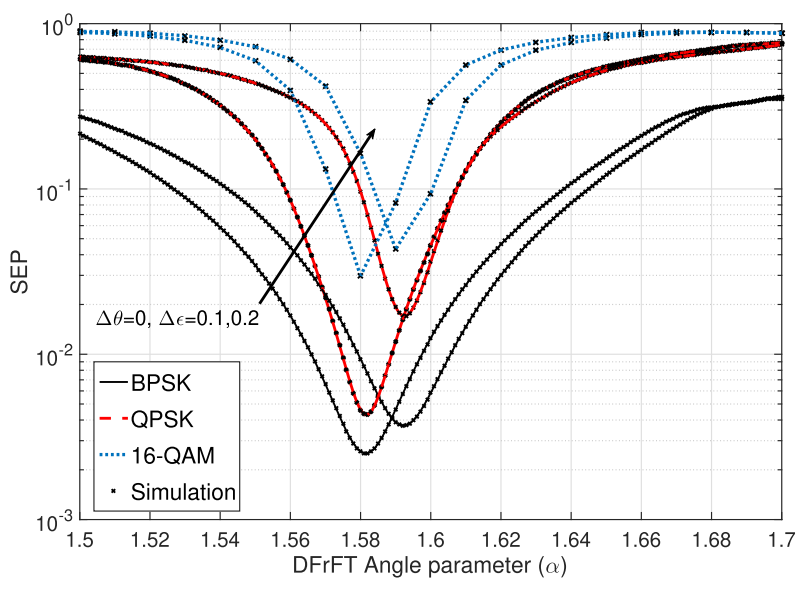

Fig. 2. Analytical and simulated SEP versus DFrFT angle parameter $\alpha$ at normalized CFO $\Delta \epsilon=0.1,0.2$ in case of 8-point DFrFT-based OFDM for BPSK, QPSK, and 16-QAM and transmission over frequency selective Rayleigh fading channel with 2-taps equal power delay profile at $\bar{\gamma}_{k}=25 \mathrm{~dB}$.

received signal used to derive all the analytical expressions of the SEP. From such an equation we observe that the first term, which denotes the desired signal, and the second term, which

$$
\begin{aligned}
& P_{s}(\xi)=\frac{3}{4}-\frac{1}{4 N 2^{4 N-2}} \sum_{k=0}^{N-1} \sum_{q=0}^{2^{N-2}-1} \sum_{l=0}^{2^{N-2}-1} \sum_{m=0}^{2^{N-2}} \sum_{n=0}^{2^{N-2}-1} \sum_{k=0}^{N-1} \sum_{f=1}^{16} \mathrm{E}_{1}^{1}(0) \Psi_{0}^{1}[1, f]\left(\frac{1}{2}+\frac{1}{\pi} \operatorname{atan}\left\{\mathrm{E}_{1}^{1}(0) \Psi_{0}^{1}[2, f]\right\}\right)+\mathrm{E}_{2}^{1}(0) \Psi_{0}^{1}[2, f] \\
& \times\left(\frac{1}{2}+\frac{1}{\pi} \operatorname{atan}\left\{\mathrm{E}_{2}^{1}(0) \Psi_{0}^{1}[1, f]\right\}\right)-\mathrm{E}_{1}^{1}(0) \Psi_{0}^{1}[1, f]\left(\frac{1}{2}+\frac{1}{\pi} \operatorname{atan}\left\{\mathrm{E}_{1}^{1}(0) \Psi_{2}^{1}[2, f]\right\}\right)-\left(\mathrm{E}_{2}^{1}(2) \Psi_{2}^{1}[2, f]\right. \\
& \times\left(\frac{1}{2}+\frac{1}{\pi} \operatorname{atan}\left\{\left(\mathrm{E}_{2}^{1}(2) \Psi_{0}^{1}[1, f]\right\}\right)-\mathrm{E}_{1}^{1}(2) \Psi_{2}^{1}[1, f]\left(\frac{1}{2}+\frac{1}{\pi} \operatorname{atan}\left\{\mathrm{E}_{1}^{1}(2) \Psi_{0}^{1}[2, f]\right\}\right)-\left(\mathrm{E}_{2}^{1}(0) \Psi_{0}^{1}[2, f]\right.\right. \\
& \times\left(\frac{1}{2}+\frac{1}{\pi} \operatorname{atan}\left\{\left(\mathrm{E}_{2}^{1}(0) \Psi_{2}^{1}[1, f]\right\}\right)+\mathrm{E}_{1}^{1}(2) \Psi_{2}^{1}[1, f]\left(\frac{1}{2}+\frac{1}{\pi} \operatorname{atan}\left\{\mathrm{E}_{1}^{1}(2) \Psi_{2}^{1}[2, f]\right\}\right)+\left(\mathrm{E}_{2}^{1}(2) \Psi_{2}^{1}[2, f]\right.\right. \\
& \times\left(\frac{1}{2}+\frac{1}{\pi} \operatorname{atan}\left\{\left(\mathrm{E}_{2}^{1}(2) \Psi_{2}^{1}[1, f]\right\}\right)+\mathrm{E}_{1}^{2}(0) \Psi_{0}^{2}[1, f]\left(\frac{1}{2}+\frac{1}{\pi} \operatorname{atan}\left\{\left(\mathrm{E}_{1}^{2}(0) \Psi_{2}^{2}[2, f]\right\}\right)+\left(\mathrm{E}_{2}^{2}(2) \Psi_{2}^{2}[2, f]\right.\right.\right. \\
& \times\left(\frac{1}{2}+\frac{1}{\pi} \operatorname{atan}\left\{\left(\mathrm{E}_{2}^{2}(a) \Psi_{0}^{2}[1, f]\right\}\right)+\mathrm{E}_{1}^{2}(2) \Psi_{2}^{2}[1, f]\left(\frac{1}{2}+\frac{1}{\pi} \operatorname{atan}\left\{\mathrm{E}_{1}^{2}(2) \Psi_{2}^{2}[2, f]\right\}\right)+\left(\mathrm{E}_{2}^{2}(2) \Psi_{2}^{2}[2, f]\right.\right. \\
& \times\left(\frac{1}{2}+\frac{1}{\pi} \operatorname{atan}\left\{\mathrm{E}_{2}^{2}(2) \Psi_{2}^{2}[1, f]\right\}\right)+\mathrm{E}_{1}^{3}(2) \Psi_{2}^{3}[1, f]\left(\frac{1}{2}+\frac{1}{\pi} \operatorname{atan}\left\{\mathrm{E}_{1}^{3}(2) \Psi_{0}^{3}[2, f]\right\}\right)+\mathrm{E}_{2}^{3}(0) \Psi_{0}^{3}[2, f] \\
& \times\left(\frac{1}{2}+\frac{1}{\pi} \operatorname{atan}\left\{\mathrm{E}_{2}^{3}(0) \Psi_{2}^{3}[1, f]\right\}\right)-\mathrm{E}_{1}^{3}(2) \Psi_{2}^{3}[1, f]\left(\frac{1}{2}+\frac{1}{\pi} \operatorname{atan}\left\{\mathrm{E}_{1}^{3}(2) \Psi_{2}^{3}[2, f]\right\}\right)-\left(\mathrm{E}_{2}^{3}(2) \Psi_{2}^{3}[2, f]\right. \\
& \times\left(\frac{1}{2}+\frac{1}{\pi} \operatorname{atan}\left\{\mathrm{E}_{2}^{3}(2) \Psi_{2}^{3}[1, f]\right\}\right)+\mathrm{E}_{1}^{4}(2) \Psi_{2}^{4}[1, f]\left(\frac{1}{2}+\frac{1}{\pi} \operatorname{atan}\left\{\mathrm{E}_{1}^{4}(2) \Psi_{2}^{4}[2, f]\right\}\right)+\mathrm{E}_{2}^{4}(2) \Psi_{2}^{4}[2, f] \\
& \times\left(\frac{1}{2}+\frac{1}{\pi} \operatorname{atan}\left\{\mathrm{E}_{2}^{4}(2) \Psi_{2}^{4}[1, f]\right\}\right) .
\end{aligned}
$$

where $\mathrm{E}_{1}^{i}(a)=\sqrt{\frac{C_{\beta(q) \beta(k)} \bar{\gamma}_{k}}{5+\bar{\gamma}_{k} \nu_{q, l, m, n}[f]+C_{\beta(k) \beta(k)} \bar{\gamma}_{k}\left(\Psi_{a}^{i}[1, f]\right)^{2}}}, \mathrm{E}_{2}^{i}(a)=\sqrt{\frac{C_{\beta(q) \beta(k)} \bar{\gamma}_{k}}{5+\bar{\gamma}_{k} \nu_{q, l, m, n}[f]+C_{\beta(k) \beta(k)} \bar{\gamma}_{k}\left(\Psi_{a}^{i}[2, f]\right)^{2}}}$,

$\Psi_{a}^{i}[1, f]=\left[D^{i}(1)+\boldsymbol{\vartheta}_{q, l, m, n}[1, f]-a\right]$ and $\Psi_{a}^{i}[2, f]=\left[D^{i}(2)+\boldsymbol{\vartheta}_{q, l, m, n}[2, f]-a\right]$.

where $\boldsymbol{D}^{1}=[11], \boldsymbol{D}^{2}=[13], \boldsymbol{D}^{3}=[31]$ and $\boldsymbol{D}^{4}=[33]$ 
is responsible for the introduction of ICI and ISI, consist of a scaling of the symbols transmitted on different sub-carriers by $S(0, k)$ and $S(l, k)$, respectively, since this function includes the effect of $\alpha, \Delta \epsilon$ and $\Delta \theta$. Therefore, all the derived expressions of SEP given in (42), (49), and (62) have the same minimum for a given value of $\Delta \epsilon$. After having observed this, one can argue that a strategy to find an expression for the optimal value of $\alpha$ consists in taking the most manageable SEP expression and look for its minimization. Hence, in our approach, we consider the SEP expression for BPSK given in (42) with respect to $\alpha$. In order to minimize $P_{s}(\xi)$ given in (42), we proceed with the calculation of the partial derivative $\partial P_{s}(\xi) / \partial \alpha$ as reported in Appendix A. The result is given in (67) at the bottom of this page. The derivation of the closed form expression of the optimal $\alpha$ can be obtained by considering the following remarks:

Remark 1: Fig. 3 reports the SEP versus $\alpha$ at $\gamma_{k}=25 \mathrm{~dB}$ with $\Delta \theta=0$ for an 8-point DFrFT-based OFDM system in case of BPSK transmission over a multi-path Rayleigh fading channel having a number of channel coefficients $L=1, \ldots, 5$ with equal power delay profile when $\Delta \epsilon=0.1,0.2$. From Fig. 3 it can be observed that the optimal $\alpha$, for a fixed $\Delta \epsilon$, is independent of channel coefficients $L$, besides being independent of the modulation scheme. With the above observation, we can say that the optimal $\alpha$ is independent of the channel co-variance, meaning that it does not depend on $z_{q}^{k}$ and $a_{q}^{k}$ and on the respective partial derivatives $\partial z_{q}^{k} / \partial \alpha$ and $\partial a_{q}^{k} / \partial \alpha$ defined in Appendix VII. Hence, the two partial derivatives $\partial z_{q}^{k} / \partial \alpha$ and $\partial a_{q}^{k} / \partial \alpha$ are set to zero in (67), which can be therefore rewritten as (68), shown at the bottom of this page. The following variables $\tilde{C}=\sqrt{f_{+}(\alpha)}, \tilde{D}=$ $\sqrt{f_{-}(\alpha)}, Z=\bar{\gamma}_{k} a_{q}^{k}+1, G G=\sqrt{A \tilde{C}^{2} / 1+A \tilde{C}^{2}+\bar{\gamma}_{k} a_{q}^{k}}$ and $F F=\sqrt{A \tilde{D}^{2} / 1+A \tilde{D}^{2}+\bar{\gamma}_{k} a_{q}^{k}}$ have been introduced in (68).

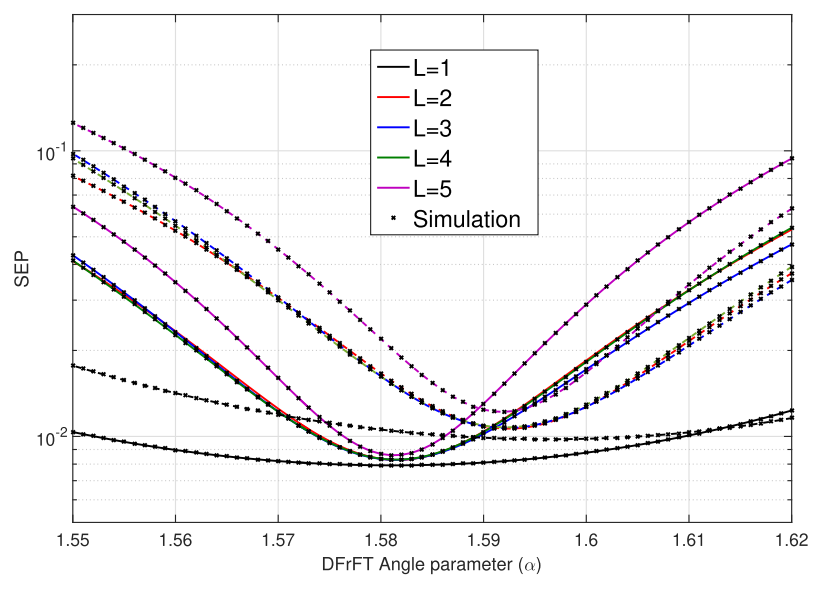

Fig. 3. Analytical and simulated SEP versus DFrFT angle parameter $\alpha$ at $\Delta \epsilon=0.1,0.2$ for 8-point DFrFT-based OFDM in the case of BPSK transmission over frequency selective Rayleigh fading channel with different values of $L$ at $\bar{\gamma}_{k}=25 \mathrm{~dB}$.

Remark 2: From Remark 1, it descends that when $\partial z_{q}^{k} / \partial \alpha$ and $\partial a_{q}^{k} / \partial \alpha$ are equal to zero it happens that $\tilde{C}=\tilde{D}$ and, therefore, we have $Y Y=Z Z$ and $W W=X X$ in (68), which can be rewritten as

$$
\frac{\partial P_{s}(\xi)}{\partial \alpha}=-\frac{2}{N 2^{N}} \sum_{q=1}^{2^{N-2}-1} \sum_{k=0}^{N-1} Y Y-\frac{2}{N 2^{N}} \sum_{q=1}^{2^{N-2}} \sum_{k=0}^{N-1} W W
$$

From (69) we observe that to have $\partial P_{s}(\xi) / \partial \alpha=0$ both the two terms at the RHS must be equal to zero individually:

- $\sum_{q=1}^{2^{N-2}-1} \sum_{k=0}^{N-1} Y Y=0$

- $\sum_{q=1}^{2^{N-2}-1} \sum_{k=0}^{N-1} W W=0$.

$$
\begin{aligned}
& \frac{\partial P_{s}(\xi)}{\partial \alpha}=-\frac{1}{N 2^{N}} \sum_{q=1}^{2^{N-2}} \sum_{k=0}^{N-1}\left\{\frac{\operatorname{sign}(\tilde{C})}{\sqrt{\frac{A \tilde{C}^{2}}{1+A \tilde{C}^{2}+\bar{\gamma}_{k} a_{q}^{k}}}} \frac{A Z \tilde{C}\left(\frac{\partial \Re[S(0, k)]}{\partial \alpha}+\frac{\partial z_{q}^{k}}{\partial \alpha}\right)-A \bar{\gamma}_{k} \tilde{C}^{2} \frac{\partial a_{q}^{k}}{\partial \alpha}}{\left(1+A \tilde{C}^{2}+\bar{\gamma}_{k} a_{q}^{k}\right)^{2}}+\sqrt{\frac{A \tilde{C}^{2}}{1+A \tilde{C}^{2}+\bar{\gamma}_{k} a_{q}^{k}}} \tilde{C} \delta(\tilde{C})\right. \\
& \times\left(\frac{\partial \Re[S(0, k)]}{\partial \alpha}+\frac{\partial z_{q}^{k}}{\partial \alpha}\right)+\frac{\operatorname{sign}(\tilde{D})}{\sqrt{\frac{A \tilde{D}^{2}}{1+A \tilde{D}^{2}+\bar{\gamma}_{k} a_{q}^{k}}}} \frac{A Z \tilde{D}\left(\frac{\partial \Re[S(0, k)]}{\partial \alpha}-\frac{\partial z_{q}^{k}}{\partial \alpha}\right)-A \bar{\gamma}_{k} \tilde{D}^{2} \frac{\partial a_{q}^{k}}{\partial \alpha}}{\left(1+A \tilde{D}^{2}+\bar{\gamma}_{k} a_{q}^{k}\right)^{2}} \\
& \left.+4 \sqrt{\frac{A \tilde{D}^{2}}{1+A \tilde{D}^{2}+\bar{\gamma}_{k} a_{q}^{k}}} \tilde{D} \delta(\tilde{D})\left(\frac{\partial \Re[S(0, k)]}{\partial \alpha}-\frac{\partial z_{q}^{k}}{\partial \alpha}\right)\right\} \\
& \frac{\partial P_{s}(\xi)}{\partial \alpha}=\frac{-1}{N 2^{N}} \sum_{q=1}^{2^{N-2}} \sum_{k=0}^{N-1}\{\underbrace{\frac{\operatorname{sign}(\tilde{C}) A Z \tilde{C}\left(\frac{\partial \Re[S(0, k)]}{\partial \alpha}\right)}{G G\left(1+A \tilde{C}^{2}+\bar{\gamma}_{k} a_{q}^{k}\right)^{2}}}_{Y Y}+\underbrace{4 G G \tilde{C} \delta(\tilde{C})\left(\frac{\partial \Re[S(0, k)]}{\partial \alpha}\right)}_{W W}+\frac{\operatorname{sign}(\tilde{D}) A Z \tilde{D}\left(\frac{\partial \Re[S(0, k)]}{\partial \alpha}\right)}{F F\left(1+A \tilde{D}^{2}+\bar{\gamma}_{k} a_{q}^{k}\right)^{2}} \\
& +\underbrace{4 F F \tilde{D} \delta(\tilde{D})\left(\frac{\partial \Re[S(0, k)]}{\partial \alpha}\right)}_{X X}\}
\end{aligned}
$$




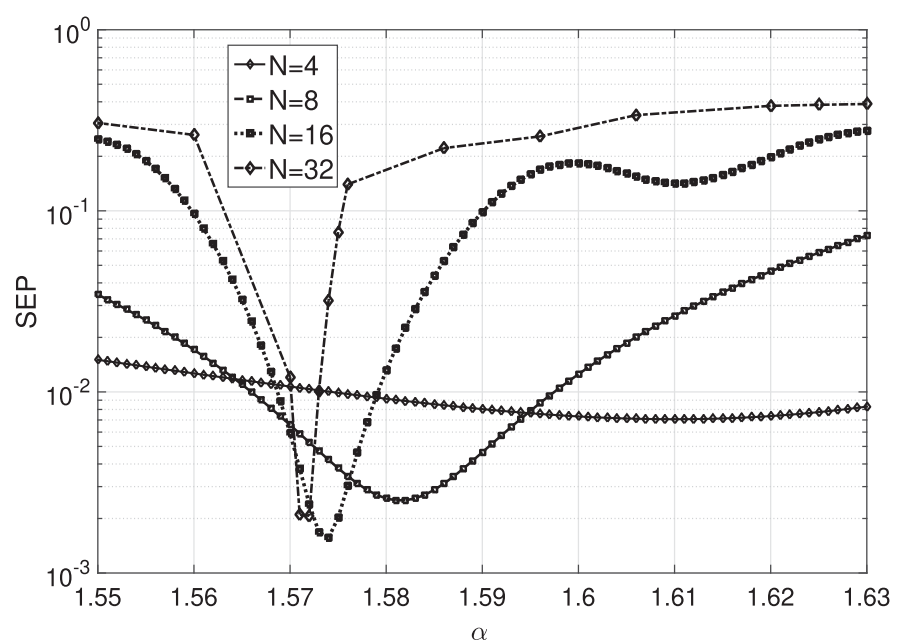

Fig. 4. Comparison of SEP versus $\alpha$ for different values of $N$ in the case of BPSK transmission over a 2-taps Rayleigh fading channel with equal power delay profile, $\Delta \epsilon=0.1$, and $\bar{\gamma}_{k}=25 \mathrm{~dB}$.

Based on both above points, this condition can be satisfied only when $\partial S(0, k) / \partial \alpha=0$.

Remark 3: In Fig. 4, we plotted the SEP versus $\alpha$ at $\bar{\gamma}_{k}=$ $25 \mathrm{~dB}$ for different values of $N$ in case of BPSK transmission over an equal power delay profile frequency selective Rayleigh fading channel with 2 taps when $\Delta \epsilon=0.1$ and $\Delta \theta=0$. From the figure it is clearly visible that the optimal $\alpha$ for fixed $\Delta \epsilon$ is a function of $N$. By using Remarks 1, 2, and 3 and following the derivation reported in the Appendix, it is now possible to compute the optimal $\alpha$ expression by setting $\partial S(0, k) / \partial \alpha=0$. After some mathematical manipulations and after substituting $\Delta \theta=0$, the final closed form expression of the optimal $\alpha$ is given by

$$
\alpha_{o p t}=10\left|\operatorname{arcCoth}\left(2 \cdot \log \left(-\exp \left(\frac{j(\Delta \epsilon)^{a} \pi}{b}\right)\right)\right)\right|,
$$

where $a=0.7 N^{0.3664}$ and $b=-25.22 N^{-0.5943}+14.62$. Finally, to confirm that the derived optimal expression for $\alpha$ gives a minimum, we computed the second partial derivative and evaluated its sign. The expression of the second order partial derivative is not reported due to the lack of space. By replacing the optimal value of $\alpha$ for different $\Delta \epsilon$ we got a positive sign, thus confirming that a minimum of SEP was achieved. The optimal $\alpha$ as a function of $\Delta \epsilon$ is reported in Fig. 7. From the results reported in the figure it is confirmed that the derived closed form expression of the optimal $\alpha$ matches with the one we are getting from the SEP performance given in Fig. 2

\section{ThEORETICAL AND SimUlation REsUlts}

In order to verify the correctness of the analysis done in Section III, SEP results obtained from Monte Carlo (MC) simulations, marked as $(x)$, are compared with those given in equations (42), (49), and (62) by considering the roll-off $\rho=0.1$. For this roll-off we verified experimentally that $N_{G}=4$ is enough for taking into account the samples that precede and follow the actual one in a particular time instant $t$. First of all, the impact of the DFrFT angle parameter $\alpha$ is numerically evaluated to find its optimal value that minimizes the SEP for different values of the normalized CFO $\Delta \epsilon$ versus $\bar{\gamma}_{k}$ with $\Delta \theta=0$. The analytical and MC simulation results, obtained for $\Delta \epsilon=0.1,0.2$ at

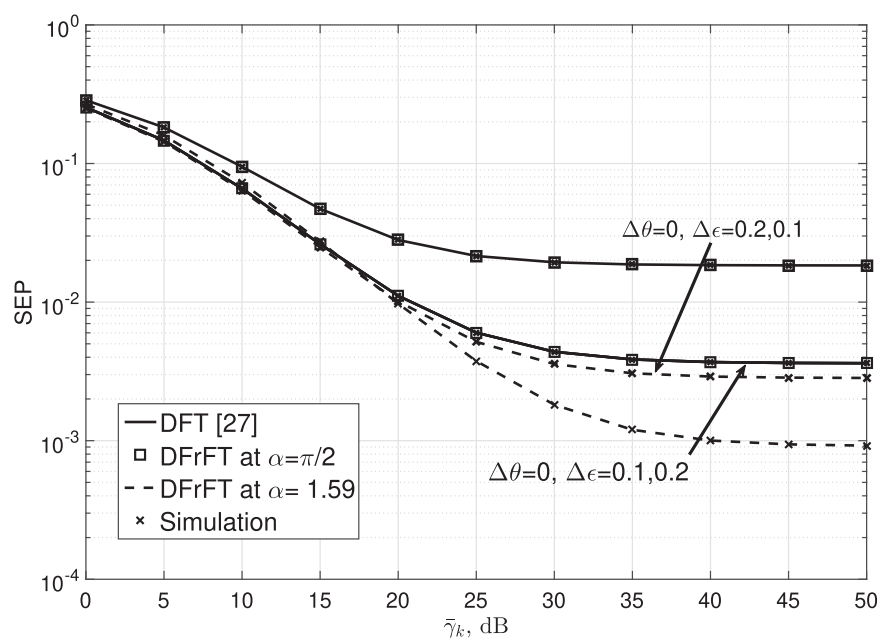

Fig. 5. Comparison of SEP versus $\bar{\gamma}_{k}$ for 8-point DFT- and DFrFT-based OFDM in case of BPSK modulation and transmission over an equal power delay 2-taps frequency selective Rayleigh fading channel at $\alpha=1.59$.

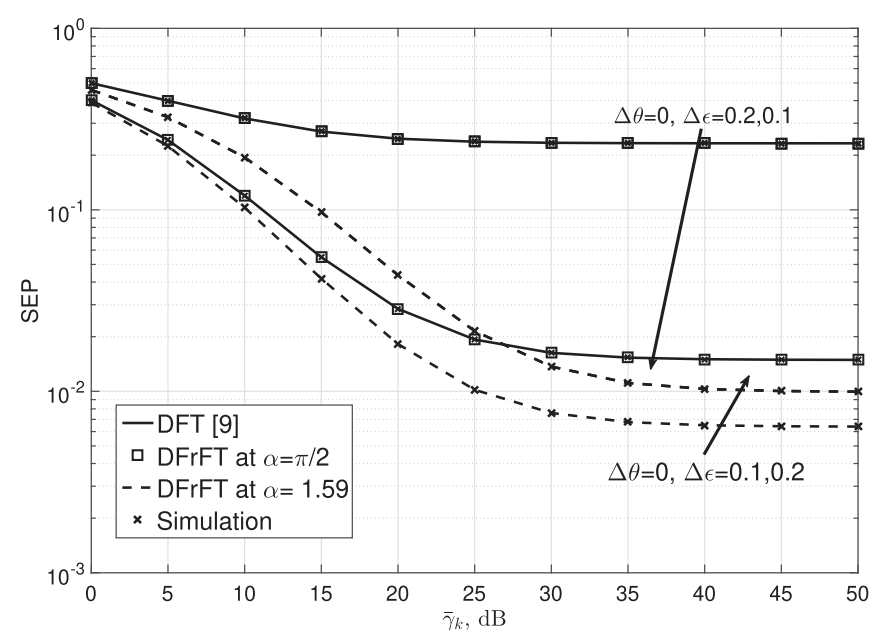

Fig. 6. Comparison of SEP versus $\bar{\gamma}_{k}$ between an 8-point DFT- and DFrFTbased OFDM system for QPSK in the case of the transmission over frequency selective Rayleigh fading channel with 2-taps of equal power delay profile at $\Delta \epsilon=0.1,0.2$ and at $\Delta \theta=0$ by considering the $\alpha=1.59$.

$\bar{\gamma}_{k}=25 \mathrm{~dB}$ with $N=8$ in case frequency selective Rayleigh fading channel with $L=2$ are shown in Fig. 2. From Fig. 2 it is clearly visible that the optimal value of $\alpha$ is independent of the modulation order for a given value of $\Delta \epsilon$. The optimal value of the angle parameter $\alpha_{o p t}$ depends only on the normalized carrier frequency offset $\Delta \epsilon$. From the figures it can be observed that simulation results confirm the derived analytical expressions. The choice of $\bar{\gamma}_{k}=25 \mathrm{~dB}$ represents a value that is about in the middle of the range of SNR values considered for measuring SEP performance, as it will be shown in Figs. 5, 6, and 8. However, the SEP performance is analyzed by considering $\alpha=1.59$ for both the two values of the CFO, $\Delta \epsilon=0.1,0.2$, and the STO $\Delta \theta=0$. Obviously, in the results presented in this section $\alpha=\pi / 2$ converts to the DFT-based OFDM. In Fig. 5 the SEP performance analysis is done in the case of BPSK modulation scheme for the DFrFT-based OFDM system with two different values of CFO, i.e., $\Delta \epsilon=0.1,0.2$. The analytical expression of the SEP given in (42) is confirmed by $\mathrm{MC}$ simulation in the case of transmission over the Rayleigh 


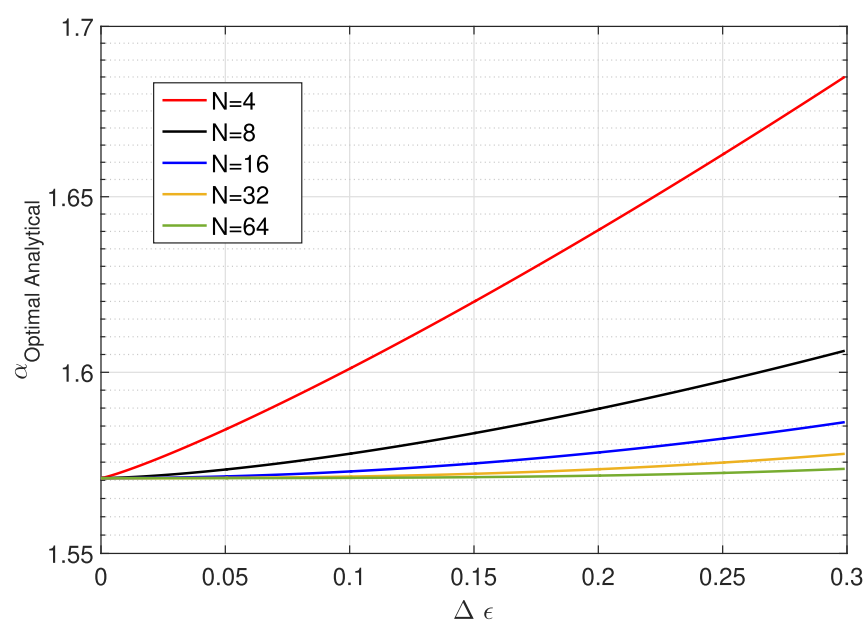

Fig. 7. Optimal DFrFT angle parameter versus $\Delta \epsilon$ for BPSK modulation DFrFT-based OFDM.

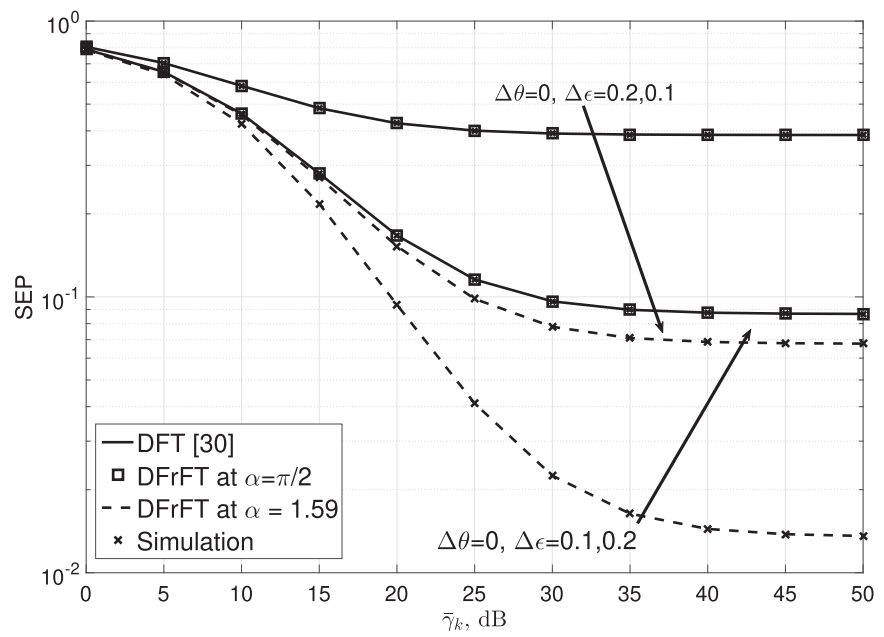

Fig. 8. Comparison of SEP versus $\bar{\gamma}_{k}$ between 8-point DFT- and DFrFTbased OFDM for 16-QAM modulation scheme transmission over frequency selective Rayleigh fading channel of 2-taps with equal power delay profile at $\Delta \epsilon=0.1,0.2$ and $\Delta \theta=0$ by considering $\alpha=1.59$.

fading channel with $L=2$. Moreover, by substituting $\alpha=\pi / 2$, the proposed expression given in (42) converts into the DFTbased OFDM and at $\alpha=1.59$ is the same for both the values of CFO, $\Delta \epsilon=0.1,0.2$. Similarly, Figs. 6 and 8 report the SEP performance for QPSK and 16-QAM, respectively, considering the same parameters used above. As it can be observed from Figs. 5, 6 , and 8 , the performance of the DFrFT-based OFDM system with $\alpha=1.59$ overcomes that of the DFT-based one and simulation results perfectly match analytical ones for different values of $\Delta \epsilon$ and $\Delta \theta=0$. Furthermore, the analysis of the STO effect on SEP performance for DFrFT-based OFDM system using the same value of the DFrFT angle parameter $\alpha=1.59$ and for $\alpha=\pi / 2$ at $\bar{\gamma}_{k}=25 \mathrm{~dB}$ is given in Figs. 9, 10, and 11. In Fig. 9 the SEP performance obtained from the analytical expression given in (42), which holds for BPSK, is plotted for different values of the CFO, $\Delta \epsilon=0.15,0.18,0.2$. From Fig. 9 , it is clearly visible that DFrFT-based OFDM system for $\alpha=1.59$ is more robust to STO than that based on DFT. Moreover, it can be observed that DFrFT-based OFDM is more robust to STO as well

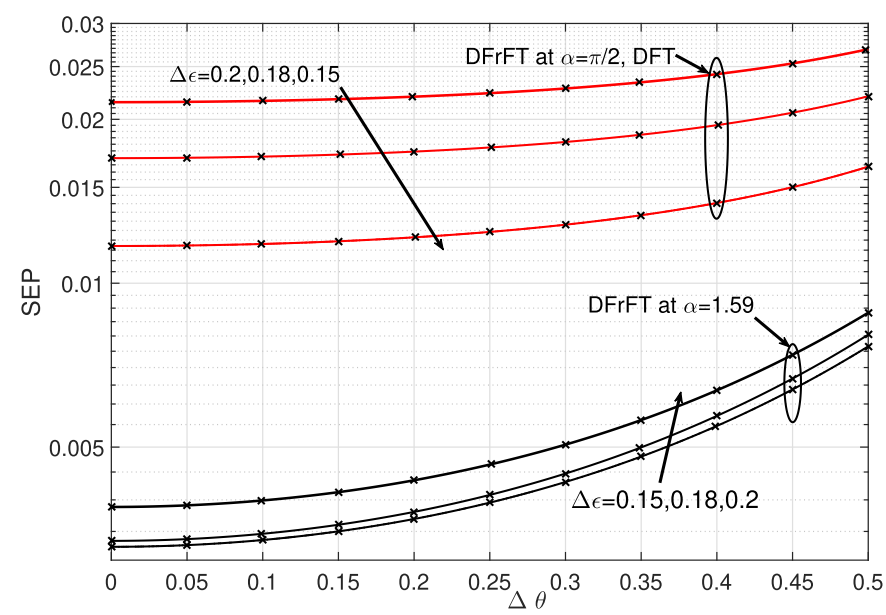

Fig. 9. Comparison of SEP versus $\Delta \theta$ between 8-point DFT- and DFrFTbased OFDM BPSK in the case of transmission over frequency selective Rayleigh fading channel with 2-taps of equal power delay profile at $\Delta \epsilon=0.15$, 0.18 , and 0.2 and $\bar{\gamma}_{k}=25 \mathrm{~dB}$ for DFrFT angle parameter $\alpha=1.59$.

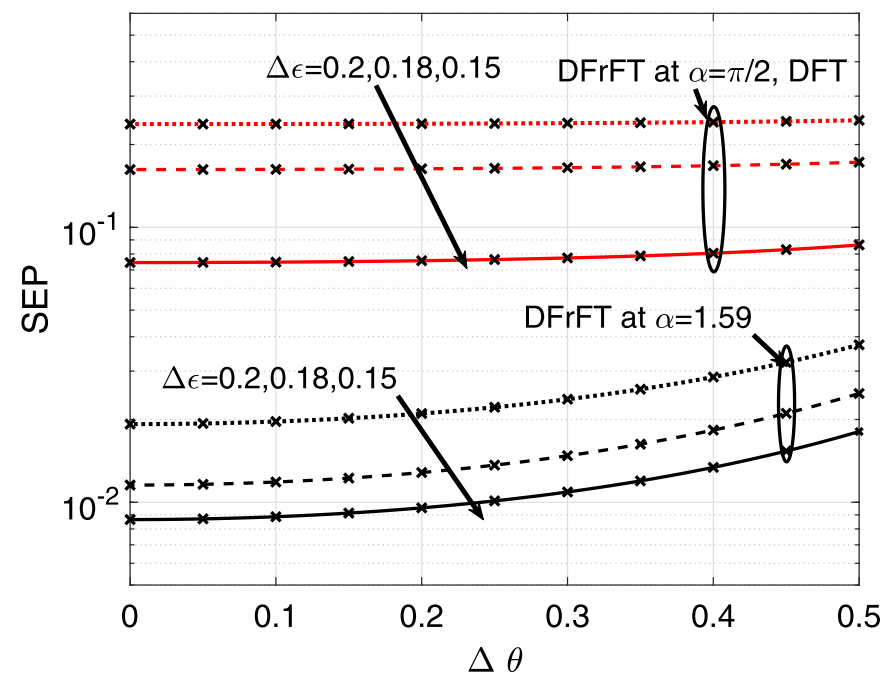

Fig. 10. Comparison of SEP versus $\Delta \theta$ between 8-point DFT- and DFrFTbased OFDM for QPSK transmission over frequency selective Rayleigh fading channel of 2-taps with equal power delay profile at normalized CFOs $\Delta \epsilon=0.15,0.18$, and 0.2 and $\bar{\gamma}_{k}=25 \mathrm{~dB}$ for DFrFT angle parameter $\alpha=1.59$.

as to CFO as compared to DFT-based OFDM. Similarly, Figs. 10 and 11 show the SEP performance of QPSK and 16-QAM, respectively, for the same values of CFO considered above. As it can be observed from Figs. 9, 10, and 11, by increasing the values of $\Delta \theta$ the SEP performance degrades significantly, the rate of degradation of DFrFT being larger as compared to that of DFT. This is because we fixed the values of the DFrFT angle parameter to $\alpha=1.59$ and evaluated over the different $\Delta \theta$. By increasing the value of $\Delta \theta$ for fixed $\alpha$ the term $S(l, k)$, which is responsible for ICI and ISI in (23), increases. Due to the fixed value $\alpha=1.59$, the rate of the degradation in SEP performance in DFrFT is larger as compared to the one based on DFT. Our results show that DFrFT-based OFDM system guarantees more robustness that DFT-based OFDM system when the effect of CFO and STO are considered together by proper selection of the DFrFT angle value $\alpha$. 


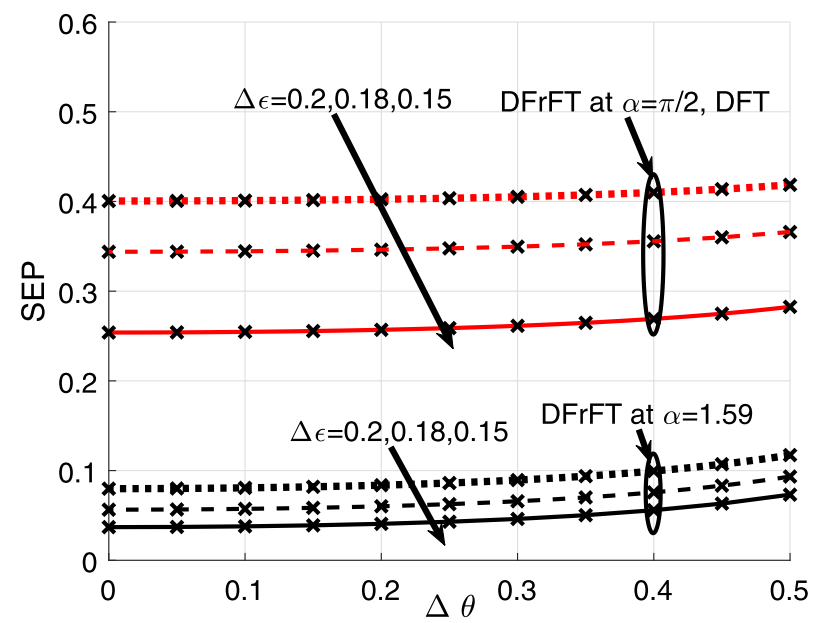

Fig. 11. Comparison of SEP versus $\Delta \theta$ between 8-point DFT- and DFrFT based OFDM for 16-QAM transmission over frequency selective Rayleigh fading channel of 2-taps with equal power delay profile at $\Delta \epsilon=0.15,0.18$ and 0.2 and at $\bar{\gamma}_{k}=25 \mathrm{~dB}$ for DFrFT angle parameter $\alpha=1.59$.

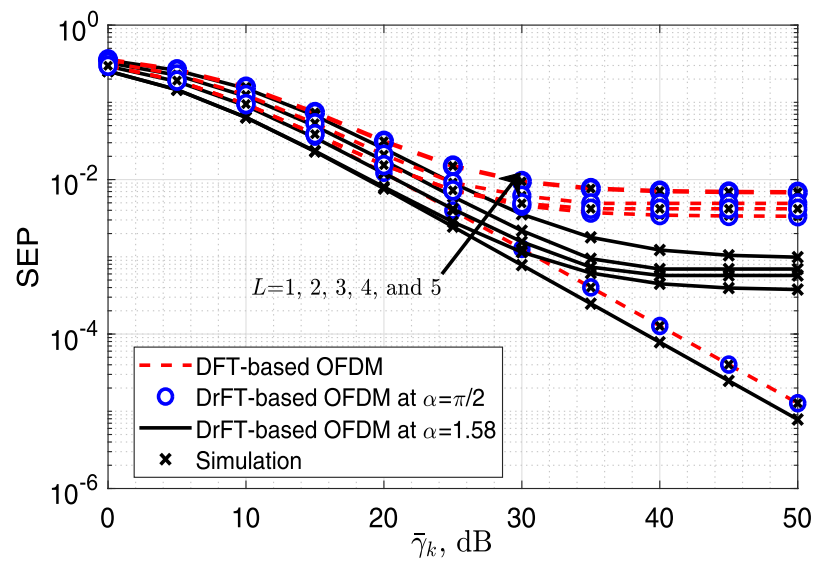

Fig. 12. Comparison of SEP versus $\bar{\gamma}_{k}$ between 8-point DFT- and DFrFT based OFDM for BPSK modulation techniques transmission over frequency selective Rayleigh fading channel with different number of channel coefficients $L$ at $\Delta \epsilon=0.1$ and $\Delta \theta=0$.

Figure 12 reports the SEP versus $\bar{\gamma}_{k}$ for different values of the multi-path Rayleigh fading channel coefficients $L$ in case of BPSK. In the figure, we compare the SEP performance for 8point DFrFT- and DFT-based OFDM system for different values of $L$ with equal power delay profile frequency selective Rayleigh fading channel when $\Delta \epsilon=0.1$ and $\Delta \theta=0$. From Fig. 12, it is clearly visible that the performance of the DFrFT-based OFDM system is always better than that of the DFT-based one for any value of $L$. Also, at $\alpha=\pi / 2$ and $\Delta \theta=0$ derived results have a perfect match with those given in literature when only CFO is present. With the same parameters of Fig. 12, in Fig. 13 a similar behavior of SEP versus $\bar{\gamma}_{k}$ is observed for QPSK. From the figure it can also be seen that at $\alpha=\pi / 2$ and $\Delta \theta=0$ there is a perfect match with the results shown in [14]. Finally, similar confirmation can be obtained for 16-QAM. The results for 16QAM are reported in Fig. 14, for which at $\alpha=\pi / 2$ and $\Delta \theta=0$ derived results there is a perfect agreement with those given in

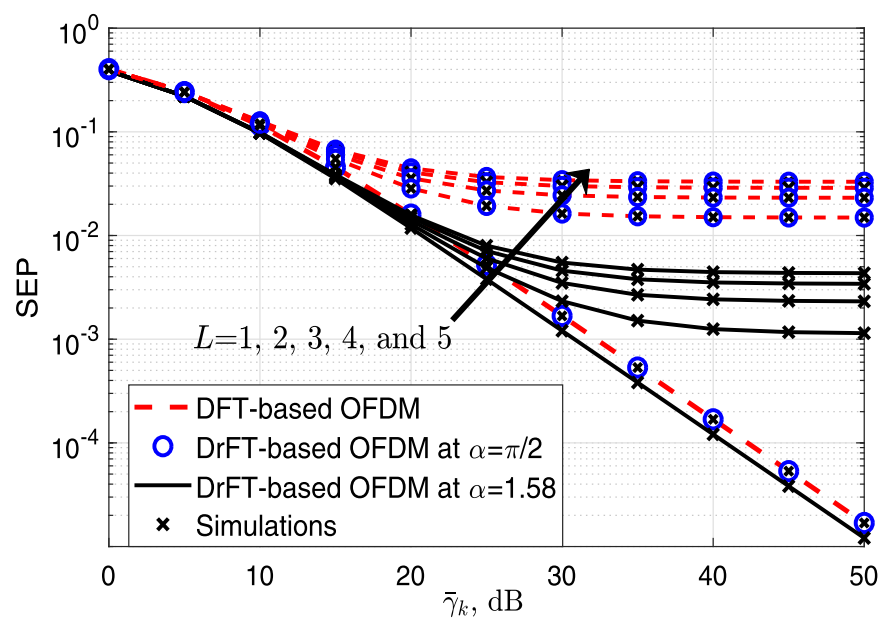

Fig. 13. Comparison of SEP versus $\bar{\gamma}_{k}$ between 8-point DFT- and DFrFT based OFDM for QPSK modulation techniques transmission over frequency selective Rayleigh fading channel with different number of channel coefficients $L$ at $\Delta \epsilon=0.1$ and $\Delta \theta=0$.

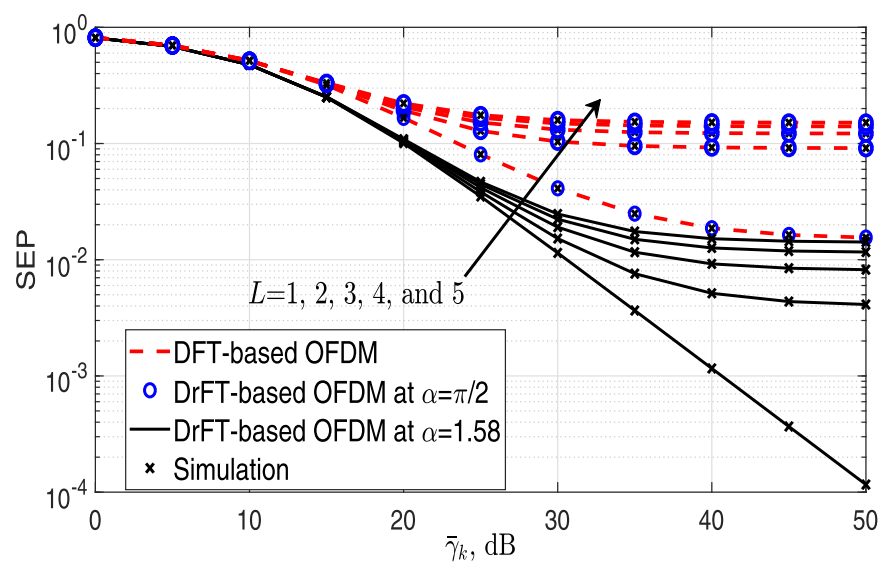

Fig. 14. Comparison of SEP versus $\bar{\gamma}_{k}$ between 8-point DFT and DFrFTbased OFDM 16-QAM transmission over frequency selective Rayleigh fading channel with different number of channel taps $L$ at $\Delta \epsilon=0.1$ and $\Delta \theta=0$.

[35]. Therefore, our results confirm the superiority of DFrFTbased OFDM compared to DFT-based OFDM when the optimal angle parameter is set for the given value of $\Delta \epsilon$.

The optimality of $\alpha$ is verified by drawing the SEP versus CFO in case of transmission over a 2-taps frequency selective Rayleigh fading channel. In Fig. 15, the SEP versus $\Delta \epsilon$ is reported by considering the fixed value of $\bar{\gamma}_{k}=25 \mathrm{~dB}$ and $\Delta \theta=0$ for an 8-point DFrFT-based OFDM system using BPSK at $\alpha=\pi / 2,1.584,1.591$, and 1.61. The chosen values of $\alpha$ are the optimal ones for $\Delta \epsilon=0.1,0.2$, and 0.3 , respectively. From Fig. 15 we observe that the best SEP performance is always better at the value of $\Delta \epsilon$ that defines $\alpha_{o p t}$ and that as we move away from both the two sides of these optimal values the performance degrades. With the same parameters given in Fig. 15, Fig. 16 reports the SEP versus $\Delta \epsilon$ for QPSK. From Fig. 16 we can observe a similar behavior in the SEP performance. Finally, the same behavior is also observed for 16-QAM from the results shown in Fig. 17. 


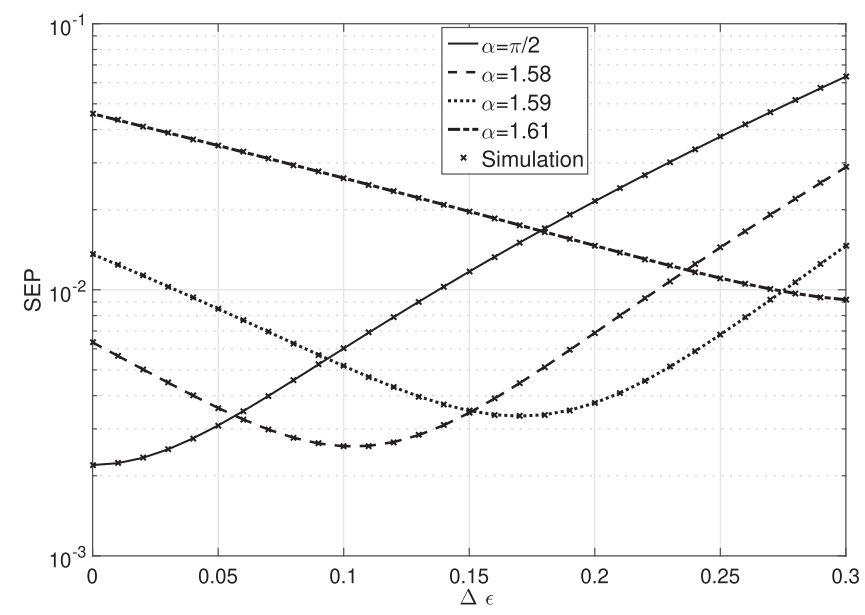

Fig. 15. Comparison of SEP versus $\Delta \epsilon$ between 8-point DFrFT-based OFDM for BPSK modulation techniques transmission over frequency selective Rayleigh fading channel with 2-taps for different $\alpha$ and at $\bar{\gamma}_{k}=25 \mathrm{~dB}$.

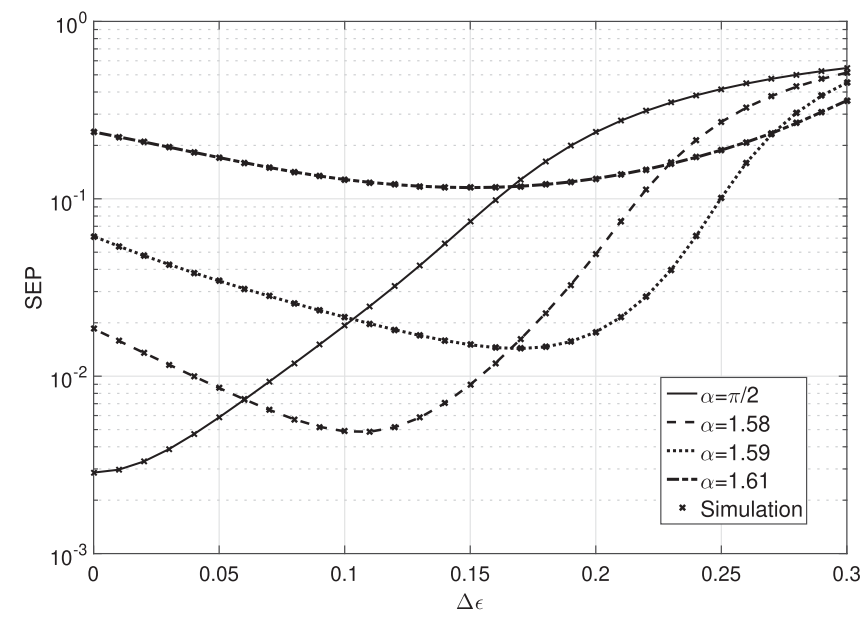

Fig. 16. Comparison of SEP versus $\Delta \epsilon$ between 8-point DFrFT-based OFDM for QPSK modulation techniques transmission over frequency selective Rayleigh fading channel with 2-taps for different $\alpha$ and at $\bar{\gamma}_{k}=25 \mathrm{~dB}$.

\section{CONCLUSION}

This article provides SEP analysis of DFrFT-based OFDM systems with CFO and STO in frequency selective Rayleigh fading channels. It is well known that DFrFT-based OFDM systems allow to improve the performance of the DFT-based one over doubly dispersive fading channels by properly choosing the optimal value of the DFrFT angle parameter $\alpha_{\text {opt }}$. In this paper we have focused on the derivation of exact SEP expressions for DFrFT-based OFDM system in case of BPSK, QPSK, and 16QAM modulation schemes for the frequency selective Rayleigh fading channel when STO and CFO are present at the same time. From the presented results, it came out for the first time that DFrFT based OFDM system is also more robust to STO $(\Delta \theta)$ as well as the CFO $(\Delta \epsilon)$ at $\alpha_{o p t}$. In order to get the confirmation of the $\alpha_{\text {opt }}$ for particular CFO values, we have also derived the closed form expression of the $\alpha_{\text {opt }}$ as a function of the $\Delta \epsilon$. The correctness of the derived analytical expressions has been demonstrated by comparing them to SEP obtained by means of Monte Carlo simulations. Our results demonstrate that at optimal value of the angle parameter $\alpha_{o p t}$ the DFrFT-based OFDM system overcomes DFT-based OFDM one because the

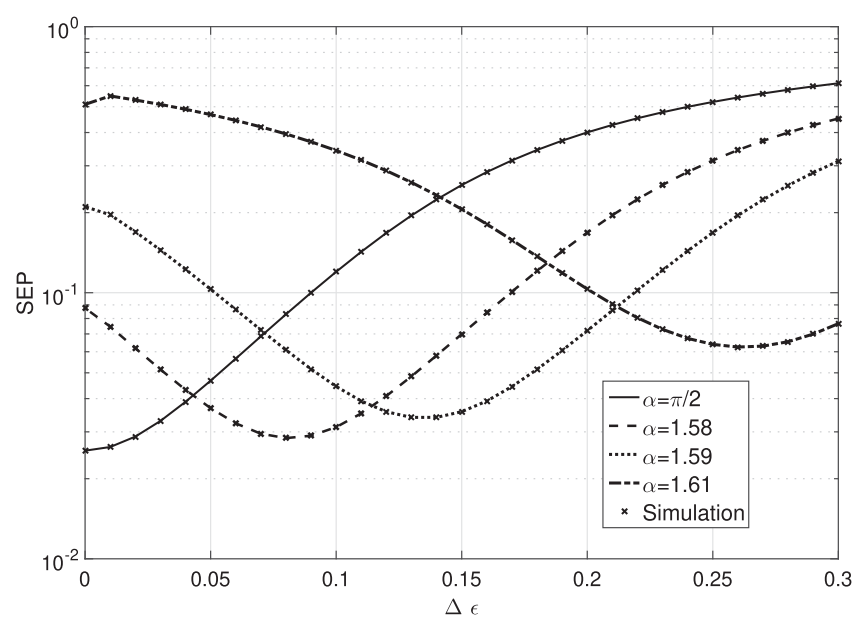

Fig. 17. Comparison of SEP versus $\Delta \epsilon$ between 8-point DFrFT-based OFDM for 16-QAM in case of transmission over frequency selective Rayleigh fading channel with 2-taps with different $\alpha$ and at $\bar{\gamma}_{k}=25 \mathrm{~dB}$.

lower sensitive to the STO as well as the CFO. For $\alpha=\pi / 2$ DFrFT-based OFDM system converts to DFT-OFDM system. A possible extension of the given SEP analysis to the massive MIMO case has been also addressed. However, an in depth analytical study of this case is left to future work.

\section{APPENDIX A \\ DERIVATIVE OF SEP EXPRESSION}

The computation of $\partial P_{s}(\xi) / \partial \alpha$ is given in (71) and shown at the top of the next page, where $P=\frac{A f_{+}(\alpha)}{1+A f_{+}(\alpha)+\bar{\gamma}_{k} a_{q}^{k}}, Q=$ $\frac{A f_{-}(\alpha)}{1+A f_{-}(\alpha)+\bar{\gamma}_{k} a_{q}^{k}}$, with $f_{+}(\alpha)=\left(\Re[S(0, k)]+z_{q}^{k}\right)^{2}$ and $f_{-}(\alpha)$ $=\left(\Re[S(0, k)]-z_{q}^{k}\right)^{2} ; A=\bar{\gamma}_{k} C_{\beta(k) \beta(k)} ; \frac{\partial f_{+}(\alpha)}{\partial \alpha}=2(\Re[S(0, k)]$ $\left.+z_{q}^{k}\right)\left(\frac{\partial \Re[S(0, k)]}{\partial \alpha}+\frac{\partial z_{q}^{k}}{\partial \alpha}\right)$, and $\frac{\partial f_{-}(\alpha)}{\partial \alpha}=2\left(\Re[S(0, k)]-z_{q}^{k}\right)$ $\left(\frac{\partial \Re[S(0, k)]}{\partial \alpha}-\frac{\partial z_{q}^{k}}{\partial \alpha}\right)$. The individual terms appearing in the above equations are defined in (72), (73)-(75), shown at the top of the next page, (76), and (77). To compute $\partial P_{s}(\xi) / \partial \alpha$, we first need to compute the derivative of the several individual terms such as $S(l, k), z_{q}^{k}$ and the $a_{q}^{k}$ with respect to $\alpha$, where $z_{q}^{k}$ and $a_{q}^{k}$ are the mean and variance of conditional random variables $b_{q}^{k} \mid \beta(k)$, respectively given in (35). To this aim, we rewrite the expression of $S(l, k)$ given in (23) as

$$
\begin{aligned}
& S(l, k)=\frac{b(l+k)}{N} \underbrace{e^{j \frac{\left(l^{2}-k^{2}\right) u^{2} \cot (\alpha)}{2}}}_{f_{4}(l, \alpha)} \\
& \underbrace{\sum_{n=0}^{N-1} e^{j \frac{2 \pi(\Delta \epsilon-l) n}{N}} e^{j \frac{n^{2} T^{2} \cot (\alpha)}{2}}}_{f_{5}(l, \alpha, \Delta \epsilon)} \\
& \underbrace{\sum_{m=0}^{N-1} e^{-j \frac{m^{2} T^{2} \cot (\alpha)}{2}} e^{j \frac{2 \pi m k}{N}}}_{f_{3}(\alpha)}, \text { for } l=0, \ldots, N-1,
\end{aligned}
$$

By using $S(l, k)$, we can also write the term $S(0, k)$ by substituting $l=0$ given in (72). Therefore, we can also rewrite the 


$$
\begin{aligned}
& \frac{\partial P_{s}(\xi)}{\partial \alpha}=-\frac{1}{N 2^{N}} \sum_{q=1}^{2^{N-2}-1} \sum_{k=0}^{N-1} \frac{\partial}{\partial \alpha}\left\{f_{1} \sqrt{P}+f_{2} \sqrt{Q}\right\}=-\frac{1}{N 2^{N}} \sum_{q=1}^{2^{N-2}-1} \sum_{k=0}^{N-1}\left\{f_{1} \frac{\partial \sqrt{P}}{\partial \alpha}+\sqrt{P} \frac{\partial f_{1}}{\partial \alpha}+f_{2} \frac{\partial \sqrt{Q}}{\partial \alpha}+\sqrt{Q} \frac{\partial f_{2}}{\partial \alpha}\right\} \\
& =-\frac{1}{N 2^{N}} \sum_{q=1}^{2^{N-2}-1} \sum_{k=0}^{N-1}\left\{f_{1} \frac{1}{2 \sqrt{p}} \frac{A \frac{\partial f_{+}(\alpha)}{\partial \alpha}\left(1+A f_{+}(\alpha)+\bar{\gamma}_{k} a_{q}^{k}\right)-A f_{+}(\alpha) \frac{\partial}{\partial \alpha}\left(1+A f_{+}(\alpha)+\bar{\gamma}_{k} a_{q}^{k}\right)}{\left(1+A f_{+}(\alpha)+\bar{\gamma}_{k} a_{q}^{k}\right)^{2}}+\sqrt{P} \frac{\partial}{\partial \alpha}\left(\operatorname{sign}\left(\sqrt{f_{+}(\alpha)}\right)\right)\right. \\
& \left.+f_{2} \frac{1}{2 \sqrt{Q}} \frac{A \frac{\partial f_{-}(\alpha)}{\partial \alpha}\left(1+A f_{-}(\alpha)+\bar{\gamma}_{k} a_{q}^{k}\right)-A f_{-}(\alpha) \frac{\partial}{\partial \alpha}\left(1+A f_{-}(\alpha)+\bar{\gamma}_{k} a_{q}^{k}\right)}{\left(1+A f_{-}(\alpha)+\bar{\gamma}_{k} a_{q}^{k}\right)^{2}}+\sqrt{Q} \frac{\partial}{\partial \alpha}\left(\operatorname{sign}\left(\sqrt{f_{-}(\alpha)}\right)\right)\right\} \\
& =-\frac{1}{N 2^{N}} \sum_{q=1}^{2^{N-2}-1} \sum_{k=0}^{N-1}\left\{\frac{f_{1}}{2 \sqrt{p}} \frac{A \bar{\gamma}_{k}\left(a_{q}^{k} \frac{\partial f_{+}(\alpha)}{\partial \alpha}-f_{+}(\alpha) \frac{\partial a_{q}^{k}}{\partial \alpha}\right)+A \frac{\partial f_{+}(\alpha)}{\partial \alpha}}{\left(1+A f_{+}(\alpha)+\bar{\gamma}_{k} a_{q}^{k}\right)^{2}}+\sqrt{P} \frac{\partial}{\partial \alpha}\left(\operatorname{sign}\left(\sqrt{f_{+}(\alpha)}\right)\right)\right. \\
& \left.+f_{2} \frac{1}{2 \sqrt{Q}} \frac{A \bar{\gamma}_{k}\left(a_{q}^{k} \frac{\partial f_{-}(\alpha)}{\partial \alpha}-f_{-}(\alpha) \frac{\partial a_{q}^{k}}{\partial \alpha}\right)+A \frac{\partial f_{-}(\alpha)}{\partial \alpha}}{\left(1+A f_{-}(\alpha)+\bar{\gamma}_{k} a_{q}^{k}\right)^{2}} \sqrt{Q} \frac{\partial}{\partial \alpha}\left(\operatorname{sign}\left(\sqrt{f_{-}(\alpha)}\right)\right)\right\}
\end{aligned}
$$

$$
\begin{aligned}
& \frac{\partial S(l, k)}{\partial \alpha}=\frac{b(k)}{N} \frac{\partial}{\partial \alpha}\left(f_{4}(l, \alpha) \cdot f_{5}(l, \alpha, \Delta \epsilon) \cdot f_{3}(\alpha)\right) \\
& =\frac{b(k)}{N}\left(e^{j \frac{\left(l^{2}-k^{2}\right) u^{2} \cot (\alpha)}{2}} \cdot \sum_{n=0}^{N-1} e^{j \frac{2 \pi(\Delta \epsilon-l) n}{N}} e^{j \frac{n^{2} T^{2} \cot (\alpha)}{2}} \cdot \sum_{m=0}^{N-1} e^{j \frac{2 \pi m k}{N}}\left(\frac{j m^{2}}{2}\left(\cot (\alpha)^{2}+1\right) e^{-\frac{j m^{2} T^{2} \cot (\alpha)}{2}}\right)+e^{j \frac{\left(l^{2}-k^{2}\right) u^{2} \cot (\alpha)}{2}}\right. \\
& \cdot \sum_{m=0}^{N-1} e^{-j \frac{m^{2} T^{2} \cot (\alpha)}{2}} e^{j \frac{2 \pi m k}{N}} \cdot \sum_{n=0}^{N-1} e^{j \frac{2 \pi(\Delta \epsilon-1) n}{N}}\left(\frac{-j n^{2}}{2}\left(\cot (\alpha)^{2}+1\right) e^{\frac{j n^{2} T^{2} \cot (\alpha)}{2}}\right)+\sum_{m=0}^{N-1} e^{-j \frac{m^{2} T^{2} \cot (\alpha)}{2}} e^{j \frac{2 \pi m k}{N}} \\
& \cdot \sum_{n=0}^{N-1} e^{j \frac{2 \pi(\Delta \epsilon-1) n}{N}} e^{j \frac{n^{2} T^{2} \cot (\alpha)}{2}} \cdot e^{-\frac{4 \pi^{2}|\sin \alpha|^{2} \cot \alpha\left(\frac{j}{2}\left(k^{2}-l^{2}\right)\right)}{N^{2}}}\left(\frac{4 \pi^{2}|\sin \alpha|^{2}\left(\cot (\alpha)^{2}+1\right)\left(\frac{j}{2}\left(k^{2}-l^{2}\right)\right)}{N^{2}}\right. \\
& \left.-\frac{8 \pi^{2}|\sin \alpha| \operatorname{sign}(\sin (\alpha)) \cos \alpha \cot \alpha\left(\frac{j}{2}\left(k^{2}-l^{2}\right)\right)}{N^{2}}\right)
\end{aligned}
$$

expressions of $z_{q}^{k}$ and $a_{q}^{k}$ as

$$
\begin{aligned}
z_{q}^{k}= & C_{\beta(k) \beta(k)}^{-1} \Re\left[\left(\boldsymbol{e}_{q}^{T} \operatorname{diag}(S(1, k), \ldots, S(N-1, k))\right)\right. \\
& \left.C_{\boldsymbol{\beta}_{k} \beta(k)}\right]
\end{aligned}
$$

and

$$
\begin{aligned}
a_{q}^{k}= & \left(\boldsymbol{e}_{q}^{T} \operatorname{diag}(S(1, k), \ldots, S(N-1, k))\right) \\
& C_{\boldsymbol{\beta}_{k} \mid \beta(k)}\left(\boldsymbol{e}_{q}^{T} \operatorname{diag}(S(1, k), \ldots, S(N-1, k))\right)^{H}
\end{aligned}
$$

The derivative of the individual terms $S(l, k), z_{q}^{k}$ and $a_{q}^{k}$ with respect to $\alpha$ are given in eqs. (75), (76) and (77) respectively, as $\frac{\partial z_{q}^{k}}{\partial \alpha}=C_{\beta(k) \beta(k)}^{-1} \Re\left[\boldsymbol{e}_{q}^{T}\left[\begin{array}{lll}\frac{\partial S(1, k)}{\partial \alpha} & & \\ & \ddots & \\ & & \frac{\partial S(N-1, k)}{\partial \alpha}\end{array}\right] C_{\boldsymbol{\beta}_{k} \beta(k)}\right]$, and

$$
\begin{aligned}
& \frac{\partial a_{q}^{k}}{\partial \alpha}=\left[\boldsymbol{e}_{q}^{T}\left[\begin{array}{lll}
\frac{\partial S(1, k)}{\partial \alpha} & & \\
& \ddots & \\
& & \frac{\partial S(N-1, k)}{\partial \alpha}
\end{array}\right]\right. \\
& C_{\boldsymbol{\beta}_{k} \mid \beta(k)}\left(\boldsymbol{e}_{q}^{T} \operatorname{diag}(S(1, k), \ldots, S(N-1, k))\right)^{H} \\
& +\left(\boldsymbol{e}_{q}^{T} \operatorname{diag}(S(1, k), \ldots, S(N-1, k))\right) \\
& \left.C_{\boldsymbol{\beta}_{k} \mid \beta(k)}\left(\boldsymbol{e}_{q}^{T}\left[\begin{array}{lll}
\frac{\partial S(1, k)}{\partial \alpha} & & \\
& \ddots & \\
& & \frac{\partial S(N-1, k)}{\partial \alpha}
\end{array}\right]\right)^{H}\right] .
\end{aligned}
$$

The result of $\partial S(l, k) / \partial \alpha$ is given in (75), for $l=0, \ldots N-1$. 


\section{REFERENCES}

[1] E. Sejdić, I. Djurović, and L. J. Stanković, "Fractional Fourier transform as a signal processing tool: An overview of recent developments," Signal Process., vol. 91, no. 6, pp. 1351-1369, Jun. 2011.

[2] Z. Mokhtari and M. Sabbaghian, "Near optimal angle of transform in FrFT-OFDM systems based on ICI analysis," IEEE Trans. Veh. Technol. vol. 65 , no. 7 , pp. 5777-5783, Jul. 2016.

[3] M. Martone, "A multicarrier system based on the fractional Fourier transform for time-frequency-selective channels," IEEE Trans. Commun., vol. 49, no. 6, pp. 1011-1020, Jun. 2001.

[4] Y. Cai, Z. Qin, F. Cui, G. Y. Li, and J. A. McCann, "Modulation and multiple access for 5G networks," IEEE Commun. Surv. Tut., vol. 20, no. 1, pp. 629-646, Mar. 2018.

[5] X. Zhang et al., "On the waveform for 5G," IEEE Commun. Mag., vol. 54, no. 11, pp. 74-80, Nov. 2016.

[6] C. Shahriar et al., "PHY-layer resiliency in OFDM communications: A tutorial," Commun. Surv. Tut., vol. 17, pp. 292-314, Mar. 2015.

[7] A. A. Zaidi et al., "Waveform and numerology to support $5 \mathrm{~g}$ services and requirements," IEEE Commun. Mag., vol. 54, pp. 90-98, Nov. 2016.

[8] B. Furht and S. A. Ahson, Eds. Long Term Evolution: 3GPP LTE Radio and Cellular Technology. Boca Raton, FL, USA: CRC Press, 2016.

[9] T. Taleb and A. Kunz, "Machine type communications in 3GPP networks: Potential, challenges, and solutions," IEEE Commun. Mag., vol. 50, no. 3 , pp. 178-184, Mar. 2012

[10] G. P. Fettweis, "The tactile Internet: Applications and challenges," IEEE Veh. Technol. Mag., vol. 9, no. 1, pp. 64-70, Mar. 2014.

[11] H. Huang, J. Yang, Y. Song, H. Huang, and G. Gui, "Deep learning for super-resolution channel estimation and DOA estimation based massive MIMO system," IEEE Trans. Veh. Technol., vol. 67, no. 9, pp. 8549-8560, Sep. 2018.

[12] O. E. Ayach, S. Rajagopal, S. Abu-Surra, Z. Pi, and R. W. Heath, Jr., "Spatially sparse precoding in millimeter wave MIMO systems," IEEE Trans. Wireless Commun., vol. 13, no. 3, pp. 1499-1513, Mar. 2014.

[13] Y. Wang and D. Xiaodai, "Comparison of frequency offset and timing offset effects on the performance of SC-FDE and OFDM over UWB channels," IEEE Trans. Veh. Technol., vol. 58, no. 1, pp. 242-250, Jan. 2009.

[14] A. M. Hamza and J. W. Mark, "Closed form SER expressions for QPSK OFDM systems with frequency offset in Rayleigh fading channels," IEEE Commun. Lett., vol. 18, no. 10, pp. 1687-1690, Oct. 2014.

[15] J. Zheng et al., "A novel timing and frequency synchronization scheme for OFDM systems," in Proc. Int. Conf. Wireless Commun., Netw. Mobile Comput., 2007, pp. 420-423.

[16] A. K. Singh and R. Saxena, "DFRFT: A classified review of recent methods with its application," J. Eng., vol. 2013, 2013, Article no. 214650, doi: 10.1155/2013/214650.

[17] S. C. Pei, C. C. Tseng, and M. H. Yeh, "A new discrete fractional Fourier transform based on constrained eigen decomposition of DFT matrix by Largrange multiplier method," IEEE Trans. Circuits Syst. II, vol. 46, no. 9, pp. 1240-1245, Sep. 1999.

[18] C. Candan, M. Kutay, and H. Ozaktas, "The discrete fractional Fourier transform," IEEE Trans. Signal Process., vol. 48, no. 5, pp. 1329-1337, May 2000.

[19] B. Santhanam and J. H. McClellan, "The discrete rotational Fourier transform,” IEEE Trans. Signal Process., vol. 44, no. 4, pp. 994-998, Apr. 1996

[20] T. Erseghe, P. Kraniauskas, and G. Cariolaro, "Unified fractional Fourier transform and sampling theorem," IEEE Trans. Signal Process., vol. 47, no. 12, pp. 3419-3423, Dec. 1999.

[21] S. C. Pei and J. J. Ding, "Closed-form discrete fractional and affine Fourier transforms," IEEE Trans. Signal Process., vol. 48, no. 5, pp. 1338-1353, May 2000.

[22] S. Liu et al., "Sparse discrete fractional Fourier transform and applications," IEEE Trans. Signal Process., vol. 62, no. 24, pp. 6582-6595, Dec. 2014.

[23] A. C. Gilbert, P. Indyk, M. Iwen, and L. Schmidt, "Recent developments in the sparse Fourier transform: A compressed Fourier transform for big data," IEEE Trans. Signal Process. Mag., vol. 31, no. 5, pp. 91-100, Sep. 2014.

[24] A. Kumar, M. Magarini, and S. Olivieri, "Rapid prototyping and FPGAin-the-Loop verification of a DFrFT-based OFDM system," in Proc. 25th Eur. Signal Process. Conf., Aug. 2017, pp. 2526-2530.

[25] S. Kumari et al., "Exact BER analysis of FRFT-OFDM system over frequency selective Rayleigh fading channel with CFO," Electron. Lett., vol. 49, no. 20, pp. 1299-1301, 2013.
[26] Z. Wang, L. Mei, X. Wang, X.Sha, and N. Zhang, "BER analysis of hybrid carrier system based on WFRFT with carrier frequency offset," Electron. Lett., vol. 51, no. 21, pp. 1708-1909, 2015.

[27] MR. Mousavi and A. Shahzadi, "Hyperbolic FRFT-OFDM system BER analysis over high Doppler Rician fading channels," Electron. Lett., vol. 51, no. 24, pp. 2003-2005, 2015.

[28] A. Kumar, M. Magarini, H. D. Joshi, and R. Saxena, "Exact SER analysis of DFrFT-based QPSK OFDM system over frequency selective Rayleigh fading channel with CFO," J. Comput. Netw. Commun., vol. 2016, 2016 , Article no. 2804507, doi: 10.1155/2016/2804507.

[29] V. Trivedi, S. Kumari, and P. Kumar, "Generalized error analysis of FRFTOFDM over Nakagami- $m$ fading channel with arbitrary $m$," IET Commun., vol. 11, no. 9, pp. 1497-1502, 2017.

[30] U. Mahesh and A. K. Chaturvedi, "Closed form BER expressions for BPSK OFDM systems with fractional timing offset and carrier frequency offset," in Proc. Nat. Conf. Commun., Kharagpur, India, 2012, pp. 1-4.

[31] M. K. Simon and M. S. Alouini, Digital Communication Over Fading Channels, 2nd ed. Hoboken, NJ, USA: Wiley, 2005.

[32] P. Dharmawansa, N. Rajatheva, and H. Minn, "An exact error probability analysis of OFDM systems with frequency offset," IEEE Trans. Commun., vol. 57, no. 1, pp. 26-31, Jan. 2009.

[33] C. Wang, J. Kim, "On the symbol error probability of 16-QAM based OFDM systems impaired by CFO," in Proc. IEEE Can. Conf. Elect. Comput. Eng., Apr. 2012, pp. 1-4.

[34] A. Annamalai, C. Tellambura, and V. K. Bhargava, "A general method for calculating error probabilities over fading channels," IEEE Trans. Commun., vol. 53, no. 5, pp. 841-852, May 2005.

[35] A. M. Hamza and J. W. Mark, "Closed-form expressions for the BER/SER of OFDM systems with an integer time offset," IEEE Trans. Commun., vol. 63, no. 11, pp. 4461-4473, Nov. 2015.

[36] R. W. Heath, N. Gonzlez-Prelcic, S. Rangan, W. Roh, and A. M. Sayeed, "An overview of signal processing techniques for millimeter wave MIMO systems," in Proc. IEEE J. Sel. Topics Signal Process., vol. 10, no. 3, pp. 436-453, Apr. 2016.

[37] W. Shen, L. Dai, B. Shim, Z. Wang, and R. W. Heath, "Channel feedback based on AoD-adaptive subspace codebook in FDD massive MIMO systems," IEEE Trans. Commun., vol. 66, no. 11, pp. 5235-5248, Nov. 2018. 